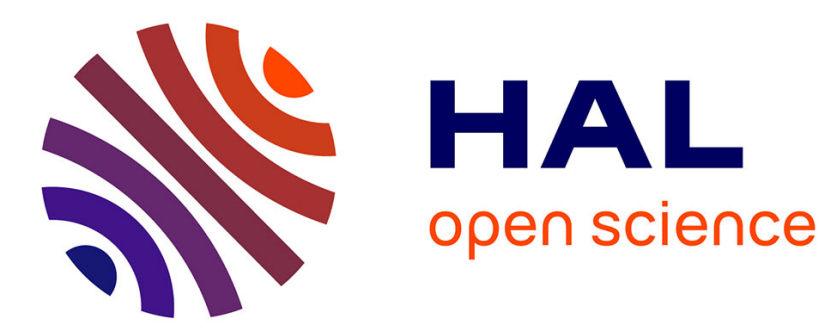

\title{
Micromorphic continuum modelling of the deformation and fracture behaviour of nickel foams
}

Thierry Dillard, Samuel Forest, Patrick Ienny

\section{To cite this version:}

Thierry Dillard, Samuel Forest, Patrick Ienny. Micromorphic continuum modelling of the deformation and fracture behaviour of nickel foams. European Journal of Mechanics - A/Solids, 2006, 25, pp.526549. 10.1016/j.euromechsol.2005.11.006 . hal-00133516

\section{HAL Id: hal-00133516 https://hal.science/hal-00133516}

Submitted on 13 Feb 2020

HAL is a multi-disciplinary open access archive for the deposit and dissemination of scientific research documents, whether they are published or not. The documents may come from teaching and research institutions in France or abroad, or from public or private research centers.
L'archive ouverte pluridisciplinaire HAL, est destinée au dépôt et à la diffusion de documents scientifiques de niveau recherche, publiés ou non, émanant des établissements d'enseignement et de recherche français ou étrangers, des laboratoires publics ou privés. 


\title{
Micromorphic continuum modelling of the deformation and fracture behaviour of nickel foams
}

\author{
T. Dillard ${ }^{\mathrm{a}}$, S. Forest ${ }^{\mathrm{a}, *}$, P. Ienny ${ }^{\mathrm{b}}$ \\ ${ }^{a}$ Ecole des Mines de Paris/CNRS, Centre des Matériaux/UMR 7633, BP 87, 91003 Evry, France \\ ${ }^{\mathrm{b}}$ Ecole des Mines d'Alès, Laboratoire de Métrologie et Mécanique, 6, av. de Clavières, 30319 Alès, France
}

\begin{abstract}
An anisotropic compressible plasticity model is incorporated into the framework of the micromorphic continuum theory in order to describe some size effects observed in ductile nickel foams. This continuum model reproduces the fact that the presence of a machined hole in a foam plate does not affect its mechanical response when the hole size becomes comparable to the cell size of the material. Finite element simulations are compared to strain field measurements in nickel foam plates with a machined hole for different hole sizes, in order to identify the characteristic length of the model. Based on a simple ductile damage law, the model is then shown to be able to account for the strong anisotropy of the initiation of crack propagation in central crack panels made of nickel foams under mode I loading conditions.
\end{abstract}

Keywords: Metallic foams; Micromorphic continuum; Compressible plasticity; Anisotropy; Finite element

\section{Introduction}

Metallic foams are strongly heterogeneous materials which are prone to strain localisation phenomena and/or size effects in their mechanical behaviour. The reason stems from the fact that the cell size can have the same order of magnitude as some characteristic structural lengths (sample size or thickness) or is comparable to the typical wavelength of variation of the applied mechanical fields. Size effects in the mechanical behaviour of metallic foams were observed for instance in notched specimens or sandwich structures (Onck et al., 2001; Andrews et al., 2001). Some hole size effects were studied in closed-cell aluminium, open-cell nickel and polymeric foams in Fleck et al. (2001). The latter paper was devoted to the study of the overall strength of these foams under compression and tension in specimens with holes of different sizes. Such effects can be attributed to two main causes:

- the intrinsic scatter of physical and mechanical properties associated with the strongly random character of the cell, strut or wall distribution and shape observed for instance in aluminium foams. The associated size effects can be modelled by appropriate statistical approaches (Blazy et al., 2004; Hohe and Becker, 2005).

\footnotetext{
* Corresponding author. Tel.: +331607630 51; fax: +33160763150.

E-mail address: samuel.forest@ensmp.fr (S. Forest).
} 
- the direct interaction between cell size and the overall mechanical fields like in the constrained deformation of metallic foams in sandwich panels (Kesler and Gibson, 2002; Chen and Fleck, 2002) or near macroscopic stress concentrations (Onck et al., 2001; Andrews et al., 2001; Fleck et al., 2001).

There is a need for continuum plasticity or damage models that are able to capture such size effects.

A rather obvious size effect is expected in the deformation of foam plates with a cylindrical machined hole of radius $R$. For large values of $R$ with respect to the cell size, stress and strain concentrations are expected at the equator according to a classical mechanical analysis of the problem. When the hole size is decreased and becomes comparable to the cell size, such a hole can hardly be distinguished from a somewhat larger cell and no systematic stress nor strain concentration is expected any longer. A continuum plasticity model, intended for calculations of structures made of metal foams, should be able to capture such a size effect and to describe the transition regime from large to small holes.

The aim of the present work is to provide an experimental quantitative field analysis of this hole size effect and to propose and calibrate a constitutive plasticity model that accounts for it at both the global (overall curves) and local levels (strain fields). The study is based on finite element analyses. It is shown then that a simple extension of this constitutive framework is able to predict some aspects of crack propagation in foam sheets.

Compressible continuum plasticity modelling was shown to be an adequate constitutive framework to treat the deformation of isotropic metallic foams in Chastel et al. (1999), Miller (2000), Deshpande and Fleck (2000). The case of plastic anisotropy was tackled for nickel foams in Badiche et al. (2000). A way of incorporating an intrinsic length scale into the continuum modelling is to resort to the mechanics of generalised continua. This approach is based on the introduction of additional degrees of freedom (rotational degrees of freedom for the Cosserat and couple stress continua, a full deformation tensor in micromorphic continua, see Eringen (1999)) or higher order gradients of the displacement field (second gradient medium, see Mindlin and Eshel (1968)). The Cosserat approach was used to replace elastic or damageable beam networks by a homogeneous equivalent medium in Pradel and Sab (1998), Onck (2002), Ebinger et al. (2005). The deformation theory of strain gradient plasticity was used in Chen and Fleck (2002) to model constrained plasticity effects in aluminium sandwich foams. An alternative continuum model is the micromorphic continuum approach which combines Cosserat and strain gradient effects in a concise and easy-to-implement framework (Eringen, 1999; Chambon et al., 2001; Forest and Sievert, 2003). The elastic-plastic micromorphic continuum was first used to model strain localisation phenomena in aluminium foams within an isotropic compressible plasticity model in Forest and Lorentz (2004), Forest et al. (2005). The model was able to regularise the simulation of the formation of horizontal strain localisation bands in foam blocks under compression, which is not the case for a pure Cosserat approach, since only limited rotation takes place within or around the band. The characteristic length of the model was directly related to the width of the strain localisation band, i.e. about the cell size.

The material investigated in this work is an open-cell nickel foam used for battery applications. It is produced in the form of foam plates of thickness $1.7 \mathrm{~mm}$. The reader is referred to the references (Ashby et al., 2000; Badiche et al., 2000) for information about material processing. The cell size is $500 \mu \mathrm{m}$ and the relative density, namely the ratio of the density of the foam and that of bulk nickel, is $\Phi^{\star}=0.035$. A complete description of the morphology of the cells and struts based on X-ray micro-tomography analyses can be found in Dillard et al. (2005). This foam displays a ductile behaviour and can be loaded in tension. An important feature of this material is the strong anisotropy of its mechanical behaviour. Three orthogonal material directions can be clearly defined after material processing: the longitudinal direction of the produced coil, called RD, the transverse direction TD and the normal direction ND. This notation is used throughout the text. The dimensions of the material along TD and RD is quasi-unlimited, whereas only 3 to 4 cells are present through the thickness along ND. In spite of this small thickness, a reproducible ductile behaviour is observed for tension along directions contained in the plane $(R D, T D)$ (Badiche et al., 2000; Dillard et al., 2005). Information on the compression behaviour with respect to the direction ND can be found in Dillard et al. (2005). The elastic properties of the foam were discussed in Badiche et al. (2000), Dillard (2004), Dillard et al. (2005). The present work concentrates on the size-dependent anisotropic plastic behaviour of nickel foams.

First, second, third and fourth rank tensors are respectively denoted by $\underline{\boldsymbol{n}}, \boldsymbol{\sim}, \underset{\sim}{\boldsymbol{K}}$ and $\underset{\sim}{\boldsymbol{C}}$ in the text. Single, double and triple contraction are written ., : and :. The gradient operator is $\nabla$. 


\section{Experimental analysis of the deformation of nickel foams}

\subsection{Anisotropic tensile behaviour}

Tensile tests were performed on nickel foam strips using an INSTRON machine with a load cell of $500 \mathrm{~N}$. Three extensometers were placed on the samples to measure the axial strain $\varepsilon_{22}$, the lateral strain $\varepsilon_{11}$ and the out-of-plane strain component $\varepsilon_{33}$. The index 2 denotes the tensile direction which will coincide either with RD or TD in the presented tests. The index 3 in tensor components always refers to the direction ND. The dimensions of the strips were:

- the width was $30 \mathrm{~mm}$, along direction 1 ;

- the length was $170 \mathrm{~mm}$, along the tensile direction 2;

- the thickness was $1.7 \mathrm{~mm}$, along direction 3 .

The obtained curves are given in Fig. 1 for tension along RD and TD directions. The tensile behaviour is characterised by an initial elastic regime followed by a short nonlinear hardening stage and finally a long almost linear hardening regime. A highly anisotropic behaviour is observed, the RD direction being more than twice stiffer than the TD one. Several tests were performed for each direction leading to a maximum scatter of $\pm 0.15 \mathrm{MPa}$ for tension along RD, and a smaller scatter along TD. Strain rates ranging from 0.000015 to $0.07 \mathrm{~s}^{-1}$ were applied, leading to slightly different stress levels which however remained within the scatter. In each test, the lateral strain components $\varepsilon_{11}$ and $\varepsilon_{33}$ were found to be very close so that only the component $\varepsilon_{11}$ is plotted in Fig. 1. The following ratio of lateral and axial strains can be defined:

$$
v_{12}^{p}=-\frac{\varepsilon_{11}^{p}}{\varepsilon_{22}^{p}} .
$$

It was found to be almost constant in the linear hardening regime of the tensile curves. The values are given in the first row of Table 1 . The corresponding values of the ratio $-\varepsilon_{33}^{p} / \varepsilon_{22}^{p}$ were also computed but are not reported here since they were very close to $v_{12}^{p}$. Again, the material exhibited strongly anisotropic lateral contraction properties. As a result, the volume changes during a tensile test are very different for tension in the RD and TD directions. For tension along $\mathrm{RD}$, the trace of the plastic strain tensor almost vanishes so that, quite astonishingly, the deformation during this test is almost isochoric. In contrast, the volume of the sample is increased by $8 \%$ at the end of the tensile test along the TD direction.

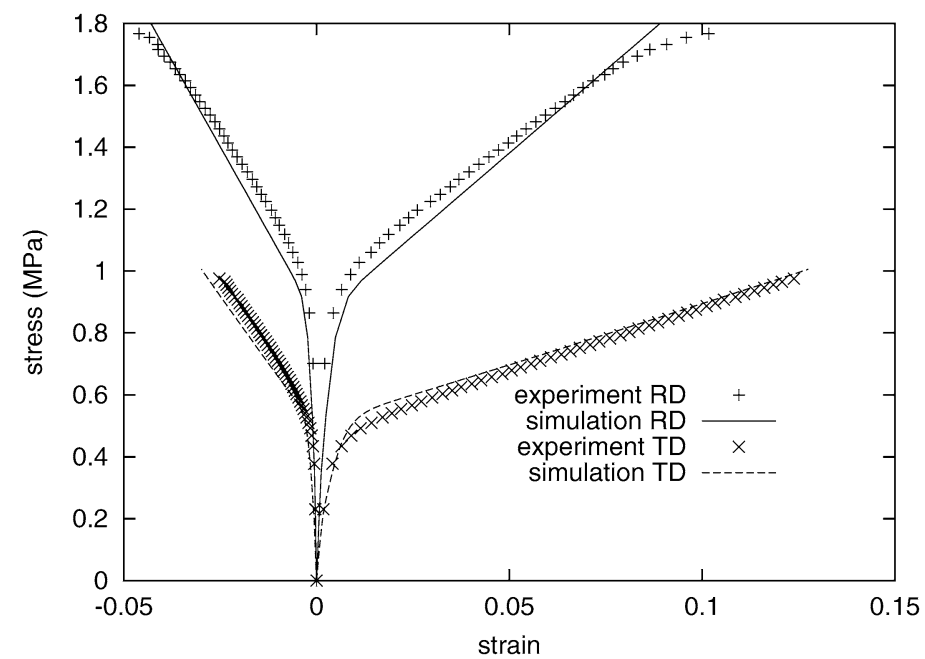

Fig. 1. Tensile behaviour of the nickel foam along the directions RD and TD. For each tensile direction, the positive strain component is the axial strain whereas the negative component is the in-plane lateral strain. Simulated results are compared with the experimental ones. 
Table 1

Lateral expansion coefficient $v_{12}^{p}$ found from point and field measurements during tensile tests on foam strips along the directions RD and TD respectively

\begin{tabular}{llr}
\hline & Direction RD & Direction TD \\
\hline Extensometers & $0.40 \pm 0.04$ & $0.19 \pm 0.02$ \\
Strain field measurement & $0.48 \pm 0.04$ & $0.17 \pm 0.02$ \\
\hline
\end{tabular}

The strong anisotropy of the observed mechanical behaviour of the nickel foam has at least two main origins (Badiche et al., 2000). The first one is the anisotropic shape of the cells in the polyurethane foam template. Secondly, the electroplated foam strip is continuously heat treated, which requires a slight tensile loading in the coiling direction to pull it through the oven. As a result of both contributions, the cells are ellipsoids with axes $a<b<c$. The axes $a, b, c$ are predominantly aligned with the directions TD, RD and ND, respectively. A detailed description of the anisotropy of cell shape and its evolution during deformation based on micro-tomographic analyses can be found in Dillard et al. (2005). A ratio $b / a=1.24$ was found in the latter work. This may explain, at least partially, why the nickel foam is harder in the direction RD than along TD.

\subsection{Strain field measurements around holes}

A strain field measurement method was applied to determine in-plane strain maps during the deformation of nickel foam sheets. Strain field measurements based on the image correlation technique are now classically used for bulk metal plates, sheets or industrial components in order to check the homogeneity of strain in basic tests like tension or to measure inhomogeneous strain fields around stress concentrators like holes or notches (Chevalier et al., 2001; Laraba-Abbes et al., 2003; Doumalin et al., 2003). Specific difficulties arise when applying this technique to metallic or polymeric foams that were first tackled in Bart-Smith et al. (1998), Bastawros et al. (2000), Badiche et al. (2000), Wang and Cuitiño (2002). They are due to the roughness and the intrinsically 3D nature of the observed surface of the foam. The local out-of-plane displacements can significantly affect the validity of the measurement of the in-plane strain components. However, as soon as the cell size becomes sufficiently small with respect to the wavelength of variation of the studied macroscopic strain fields, the method can provide quantitatively accurate results. In Badiche et al. (2000) for instance, it was shown that finite element simulations results based on an anisotropic compressible continuum plasticity model compare well with strain field measurements around holes of diameter $25 \mathrm{~mm}$. In the present work, we analyse the results of such strain field measurements in the case of holes of decreasing radii.

The strain field measuring system was made of a CCD camera providing $1280 \times 1024$ pixel images with 256 grey levels, and of an image acquiring system on a PC (Pentium III, $866 \mathrm{MHz}$ ). A $35 \mathrm{~mm}$ to $70 \mathrm{~mm}$ camera lens was used. The camera was at a fixed position at a distance of $980 \mathrm{~mm}$ from the specimen. Since the sample underwent only relatively low strain levels, a sufficiently large part of the sample remains in the image during the entire test. Black paint is spread over the samples to avoid light reflection. The rough surface naturally induces fluctuating grey levels on the initial image that are sufficient to apply a correlation algorithm to the subsequent images and retrieve with remarkable confidence selected points at different deformation stages.

During the mechanical test, images of the initial and deformed states of a part of the foam strip were recorded. An image correlation algorithm was then applied that ultimately provided values of the displacement components $u_{1}$ and $u_{2}$ at the nodes of a virtual regular grid of points selected on the initial image (see Fig. 2). The following parameters determine the precision and scatter of the estimated displacements:

- The image resolution is the physical size on the sample represented by each square pixel. Resolutions ranging from 15 to $500 \mu \mathrm{m} /$ pixel were initially tested. A resolution of $150 \mu \mathrm{m} /$ pixel was finally chosen for the tests presented in this work.

- The grid step $G_{s}$ characterises the lattice of points selected in the initial image and for which the displacements are determined. A regular square grid is chosen. Grid steps ranging from 1 to 15 pixels were tested and a value of $G_{s}=1$ was used for the results of this work.

- The correlation size $C_{s}$ is the dimension of a square window centred on the point that one wants to follow during deformation. The grey levels inside this window represent the pattern which the correlation algorithm will look for in the subsequent images. The program looks for similar patterns around the considered point in the subsequent 


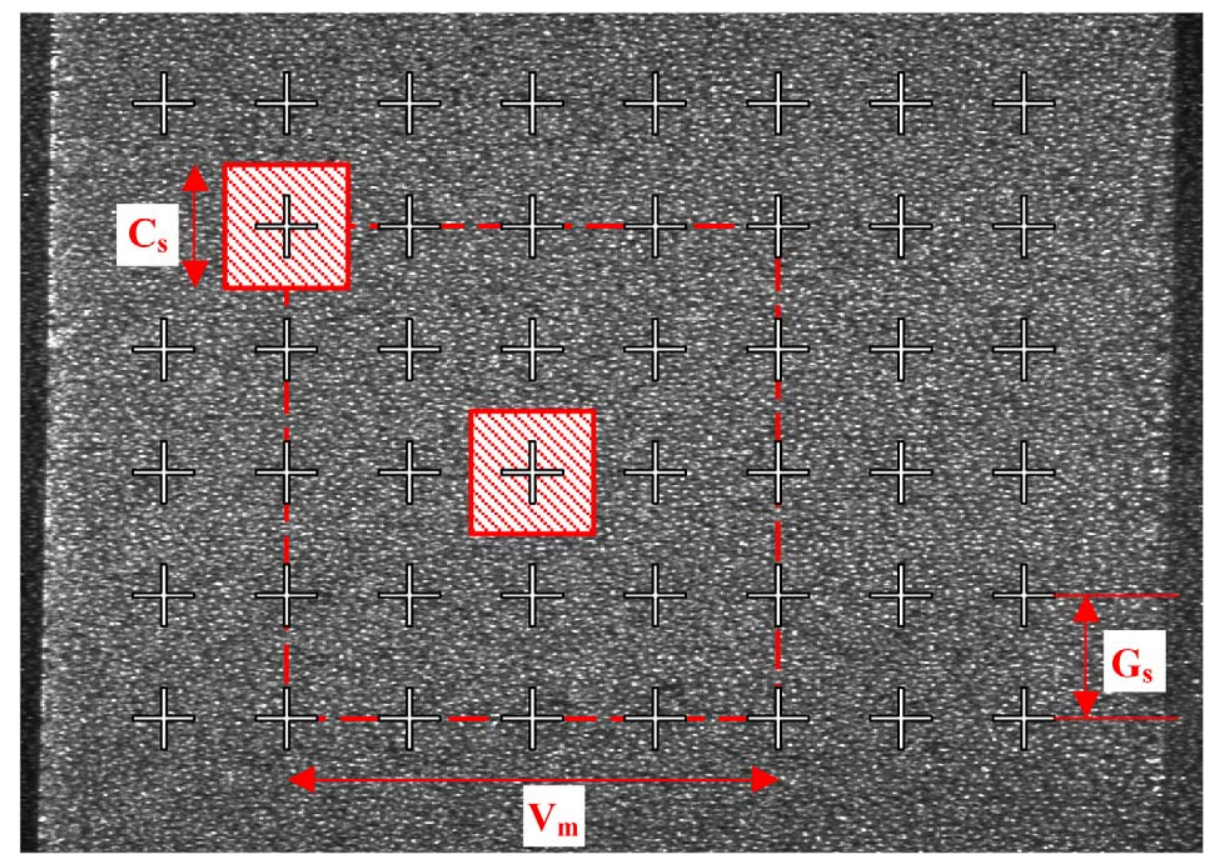

Fig. 2. Parameters of the image correlation algorithm. A virtual grid is selected on the initial image of the specimen.

image within a zone characterised by the dimension $V_{m}$ (see Fig. 2). Window sizes from $3 \times 3$ to $50 \times 50$ pixels were tested. A final correlation size of $C_{s}=30$ pixels was selected for the results presented in this work. Maximal exploration zones $V_{m}$ corresponding to $4 C_{s}$ are sufficient.

- The strain interpolation size $N_{s}$ is used to estimate the strain components at a given point of the grid from the knowledge of the displacement components of the nodes contained in the $N_{s} \times N_{s}$ window around the considered point. The gradient of the displacement field is estimated at each node in this window based on first neighbour approximation. The strain field within the $N_{s} \times N_{s}$ window is then approximated by a fifth order polynomial. $N_{s}=5$ was used in this work.

The mentioned parameters are such that significantly smaller values lead to highly fluctuating strain values and significantly higher values smooth out the strain gradients. According to this choice, the value of a strain at a given point is affected by the values of surrounding points in $3 \times 3$ cells. The displacements are estimated with a maximum error of $6 \mu \mathrm{m}$. The relative precision in the estimation of strain components for the whole apparatus was found to be 0.0016 (Dillard, 2004).

The method was first applied to nickel foam strips in tension without any hole to check the homogeneity of their deformation and the values of lateral expansion coefficients found from point measurements (see Section 2.1). The axial and lateral strain components were found to display some heterogeneities along the gauge length. These fluctuations which can reach $20 \%$ with respect to the mean strain do not lead to strain localisation during the tensile test. This has already been observed in Badiche et al. (2000). These fluctuations can be related to the rather small thickness of the material which corresponds to only 3 cells in average. Fluctuations of the actual thickness may lead to fluctuations of the strain field during tension. The strain fields $\varepsilon_{11}$ and $\varepsilon_{22}$ can be averaged over the whole sample and compared to the values found using standard extensometers. A good agreement between both types of measurements can be seen in Table 1.

The method was then applied to the analysis of the strain field around a cylindrical circular hole with radius $R$ machined in a foam plate. The holes were either stamped, saw-cut or electro-eroded in the foam plates. No significant effect of the machining was detected in the results. The investigated radii were $R=4 \mathrm{~mm}, R=2 \mathrm{~mm}, R=1 \mathrm{~mm}$. Results obtained for $R=12 \mathrm{~mm}$ were already reported in Badiche et al. (2000). The width of the foam plates were 40 or $20 \mathrm{~mm}$. The length was $170 \mathrm{~mm}$. The plates with a hole are then subjected to an overall tensile test along the TD direction. The test is displacement-controlled. Three tests were carried out for each hole size in order to check 

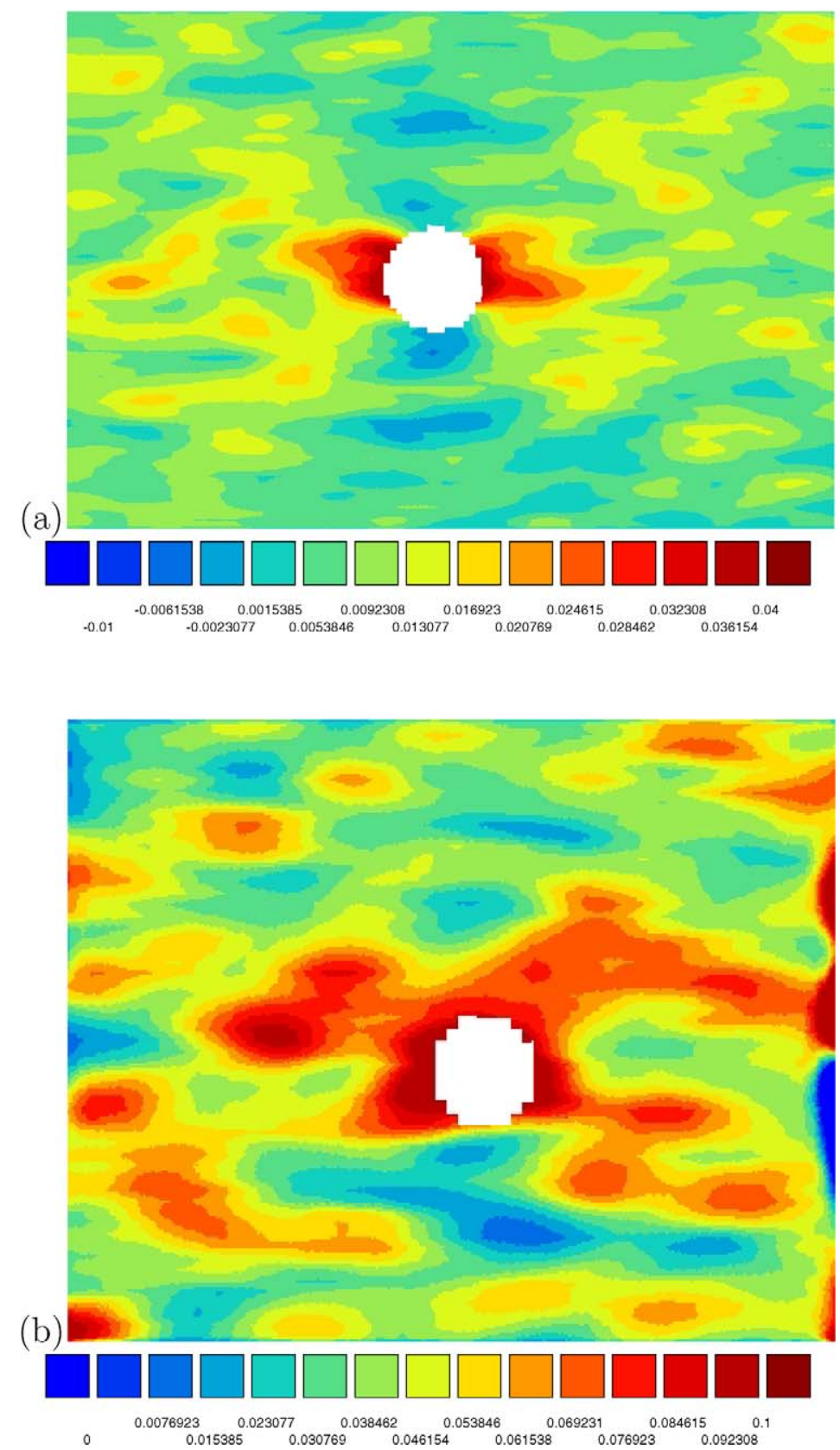

Fig. 3. Strain field $\varepsilon_{22}$ around a hole in a nickel foam plate as determined from image correlation: (a) hole radius $R=2$ mm (specimen width: $40 \mathrm{~mm}$, overall loading strain $\varepsilon_{22}^{\infty}=0.01$ ), (b) hole radius $R=1 \mathrm{~mm}$ (specimen width: $20 \mathrm{~mm}$, overall loading strain $\varepsilon_{22}^{\infty}=0.05$ ). The plate is subjected to tension along the vertical axis 2 which is parallel to TD in this test.

the reproducibility. Again, local fluctuations of $10 \%$ to $20 \%$ were found in the measured strain values at the same positions from a sample to another.

The strain fields $\varepsilon_{22}$ deduced from image correlation analysis at a given remote axial tensile strain $\varepsilon_{22}^{\infty}$ are shown in Fig. 3 in the case $R=2 \mathrm{~mm}$ and $R=1 \mathrm{~mm}$. The strain fields obtained for $R=4 \mathrm{~mm}$ are similar to that found in the case $R=2 \mathrm{~mm}$. The tensile direction is vertical in Fig. 3. The strain concentration at the equator of the hole $R=2 \mathrm{~mm}$ is clearly visible in Fig. 3(a). In contrast, there is no clear strain concentration around the hole $R=1 \mathrm{~mm}$. Instead, strain fluctuations over the whole zone are observed that are similar to the band-like structures of heterogeneity 


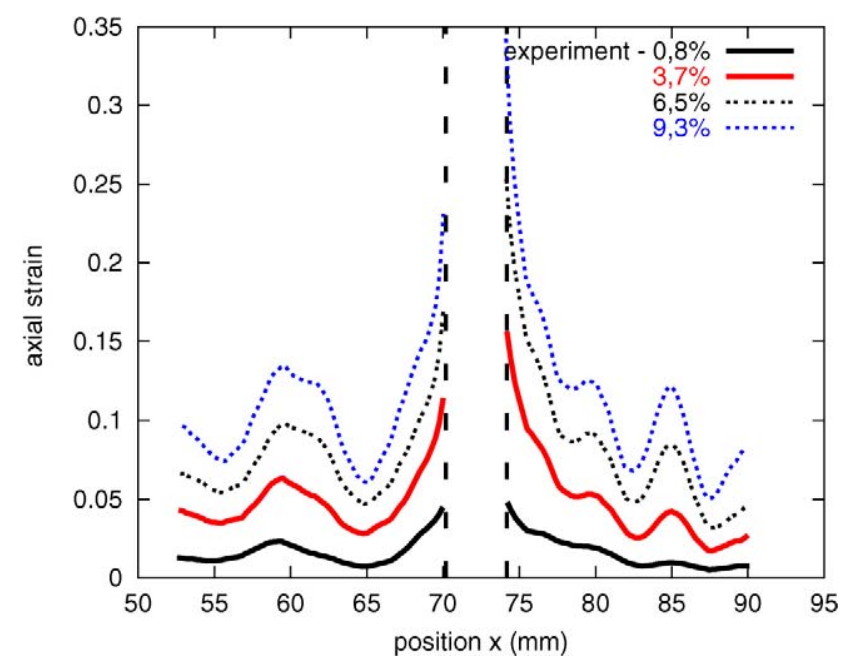

(a)

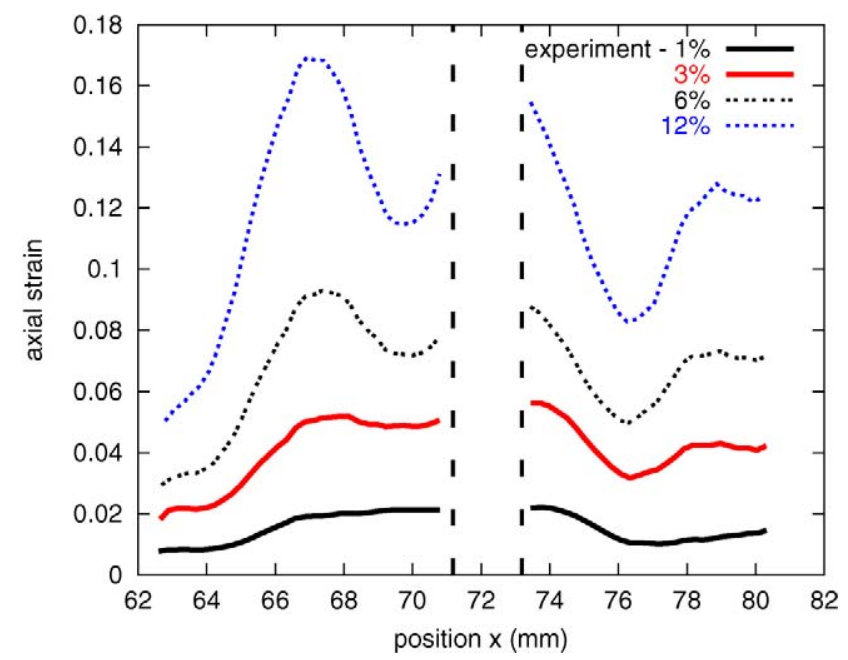

(b)

Fig. 4. Strain component $\varepsilon_{22}$ along the ligament of the nickel foam plate with a machined hole (coordinate axis $x_{1}$ ) from the strain field measurements at different overall strain levels $\varepsilon_{22}^{\infty}$ for two different hole radii. The dotted lines denote the position of the hole. (a) $R=2 \mathrm{~mm}$ (specimen width: $40 \mathrm{~mm}$ ); (b) $R=1 \mathrm{~mm}$ (specimen width: $20 \mathrm{~mm}$ ). The tensile loading direction is TD.

observed in nickel foam strips in tension in Badiche et al. (2000). The amplitude of the strain fluctuations are of the order of \pm 0.02 (Fig. 3(b)). This can be seen in a more quantitative way when the strain component $\varepsilon_{22}$ is plotted along the ligament, i.e. the horizontal line going through the equator of the hole. For the $R=2 \mathrm{~mm}$ hole, the strain profiles are shown at different overall strain levels $\varepsilon_{22}^{\infty}$ in Fig. 4(a). Strain concentration factors of about 2 to 3 are observed at the equator. Such a strain concentration factor is defined as the ratio of the axial strain at the equator and the axial strain $\varepsilon_{22}^{\infty}$ far from the hole. The local strain fluctuations observed on foam strips without any machined hole and studied in Badiche et al. (2000) are still present but the strain concentration due to the hole can be clearly distinguished from these fluctuations. In contrast, Fig. 4(b) shows that the situation is quite different for a $R=1 \mathrm{~mm}$ hole: no actual strain concentration is observed close to the equator. The amplitude of strain variations along the ligament is significantly smaller than for the $R=2 \mathrm{~mm}$. Strain concentrations around $R=1 \mathrm{~mm}$ holes, if they exist at all, are then below the strain measurement precision. In any case, they cannot be large since the final crack leading to the fracture of the samples with hole size $R=1 \mathrm{~mm}$ did not systematically initiate at the machined hole ( 1 out of three samples failed with a crack starting from the hole), in contrast to the samples with $R=4 \mathrm{~mm}$ and $R=2 \mathrm{~mm}$. 
The strain field measurements show that there is, as expected, a transition from the regime for which a hole in a foam must be regarded as a macroscopic defect, to the regime for which a machined hole cannot be distinguished from a merely larger cell. This transition takes place at hole sizes of about $R=1 \mathrm{~mm}$. This characteristic size can be compared to the mean cell diameter of $500 \mu \mathrm{m}$ but also to the fluctuation of cell sizes that is observed in such a nickel foam. These morphological parameters were investigated in Dillard et al. (2005) via X-ray microtomography. The cells are in fact ellipsoid-like with a ratio of the largest axis divided by the smallest equal to $c / a=1.5: 2 c=0.632 \pm 0.078 \mathrm{~mm}$, $2 a=0.420 \pm 0.032 \mathrm{~mm}$.

\section{Continuum plasticity modelling of nickel foams}

The constitutive equations of a continuum anisotropic compressible plasticity model introduced in Badiche et al. (2000) are first recalled. They are then extended to incorporate intrinsic length scales within the framework of a micromorphic continuum.

\subsection{Anisotropic compressible plasticity model}

Within the context of small deformation, the total strain is divided into elastic and plastic parts:

$$
\underset{\sim}{\varepsilon}={\underset{\sim}{\varepsilon}}^{\mathrm{e}}+{\underset{\sim}{\boldsymbol{\varepsilon}}}^{\mathrm{p}}, \quad \underset{\sim}{\sigma}=\underset{\sim}{\boldsymbol{C}}:{\underset{\sim}{\varepsilon}}^{\mathrm{e}}
$$

where $\underset{\widetilde{\alpha}}{\boldsymbol{C}}$ is the four-rank tensor of elastic moduli. The yield function $f(\underset{\sim}{\sigma}, R)$ includes the influence of the deviatoric part $\sigma_{\sim}^{\text {dev }}$ and of a weighted trace of the stress tensor:

$$
f\left(\underset{\sim}{\boldsymbol{\sigma}}, \sigma_{Y}\right)=\sigma_{\mathrm{eq}}-\sigma_{Y}, \quad \sigma_{\mathrm{eq}}=\left(\frac{3}{2} C \underset{\sim}{\boldsymbol{\sigma}^{\mathrm{dev}}}: \underset{\sim}{\boldsymbol{H}}: \underset{\sim}{\underset{\sim}{\boldsymbol{\sigma}} \mathrm{dev}}+F(\underset{\sim}{\boldsymbol{P}}: \underset{\sim}{\boldsymbol{\sigma}})^{2}\right)^{1 / 2} .
$$

The previous expression includes two tensors of material parameters characterising the plastic anisotropy. The corresponding matrices are written as follows in the coordinate frame associated with the symmetry axes of the foam sheet $(R D, T D, N D)$ :

$$
\underset{\sim}{\boldsymbol{H}}]=\left[\begin{array}{cccccc}
H_{a} & 0 & 0 & 0 & 0 & 0 \\
0 & H_{b} & 0 & 0 & 0 & 0 \\
0 & 0 & H_{c} & 0 & 0 & 0 \\
0 & 0 & 0 & H_{d} & 0 & 0 \\
0 & 0 & 0 & 0 & H_{e} & 0 \\
0 & 0 & 0 & 0 & 0 & H_{f}
\end{array}\right], \quad[\underset{\sim}{\boldsymbol{P}}]=\left[\begin{array}{ccc}
P & 0 & 0 \\
0 & Q & 0 \\
0 & 0 & R
\end{array}\right] .
$$

The matrix associated with $\underset{\sim}{\boldsymbol{H}}$ acts on the column formed by the six components of the stress tensor $\left[\begin{array}{llll}\sigma_{11}^{\mathrm{dev}} & \sigma_{22}^{\mathrm{dev}} & \sigma_{33}^{\mathrm{dev}}\end{array}\right.$ $\left.\begin{array}{lll}\sigma_{12}^{\mathrm{dev}} & \sigma_{23}^{\mathrm{dev}} & \sigma_{31}^{\mathrm{dev}}\end{array}\right]^{\mathrm{T}}$. The components of the matrix $[\underset{\sim}{\boldsymbol{P}}]$ are used to compute $\underset{\sim}{\boldsymbol{P}}: \underset{\sim}{\sigma}=P_{i j} \sigma_{i j}$.

When the material parameter $F=0$, the yield function reduces to Hill's criterion used for the anisotropic plasticity of dense metals (Besson et al., 2001). When $\underset{\sim}{\boldsymbol{P}}=\underset{\sim}{\mathbf{1}}$ (second-order identity tensor), the invariant quantity $\underset{\sim}{\boldsymbol{P}}: \underset{\sim}{\boldsymbol{\sigma}}$ is nothing but the trace of the stress tensor. When $F \neq 0, \underset{\widetilde{\sim}}{\boldsymbol{H}} \underset{\sim}{\mathbf{1}}, \underset{\sim}{\boldsymbol{P}}=\underset{\sim}{\mathbf{1}}$, the yield criterion reduces to the isotropic elliptic criterion classically used in powder metallurgy for instance Besson and Abouaf (1989) and also applied recently to metallic foams in Chastel et al. (1999), Deshpande and Fleck (2000).

Associative plasticity, with the direction of plastic straining normal to the yield surface, is assumed to hold for metallic foams, as done in Chastel et al. (1999), Miller (2000), Deshpande and Fleck (2000). It leads to the following expression of the plastic flow:

$$
\dot{\sim}^{\mathrm{p}}=\dot{p} \underset{\sim}{\boldsymbol{N}}, \quad \underset{\sim}{\boldsymbol{N}}=\frac{1}{\sigma_{\mathrm{eq}}}\left(\frac{3}{2} C\left(\underset{\sim}{\boldsymbol{H}}:{\underset{\sim}{\boldsymbol{\sigma}}}^{\mathrm{dev}}\right)^{\mathrm{dev}}+F(\underset{\sim}{\boldsymbol{P}}: \underset{\sim}{\boldsymbol{\sigma}}) \underset{\sim}{\boldsymbol{P}}\right)
$$

where $\dot{p}$ is the plastic multiplier. The latter can be computed from the consistency condition $\dot{f}=0$ under plastic loading (Besson et al., 2001):

$$
\dot{p}=\frac{\underset{\sim}{\boldsymbol{N}}: \underset{\sim}{\boldsymbol{C}: \underset{\sim}{\boldsymbol{\varepsilon}}}: \underset{\sim}{\boldsymbol{N}}+\mathrm{d} \sigma_{Y} / \mathrm{d} p}{\underset{\sim}{\boldsymbol{N}} .}
$$


Table 2

Material parameters of the anisotropic compressible plasticity model identified for a nickel foam with relative density $\Phi^{\star}=0.035$

\begin{tabular}{lllllllllll}
\hline $\begin{array}{l}C_{11} \\
(\mathrm{MPa})\end{array}$ & $\begin{array}{l}C_{12} \\
(\mathrm{MPa})\end{array}$ & $\begin{array}{l}C_{22} \\
(\mathrm{MPa})\end{array}$ & $\begin{array}{l}C_{44} \\
(\mathrm{MPa})\end{array}$ & & & & & & & \\
\hline 464 & 172 & 239 & 105 & & & & & & \\
\hline$C$ & $F$ & $P$ & $Q$ & $R$ & $H_{a}$ & $H_{b}$ & $H_{c}$ & $H_{d}$ & $H_{e}$ & $H_{f}$ \\
\hline 1 & 0.000682 & 1 & 36.6 & 22.6 & 0.41 & 1.8 & 1.75 & 1.49 & 1 \\
\hline $\begin{array}{l}\sigma_{0} \\
(\mathrm{MPa})\end{array}$ & $Q_{1}$ & $b_{1}$ & $\begin{array}{l}Q_{2} \\
(\mathrm{MPa})\end{array}$ & & $b_{2}$ & & & & \\
\hline 0.3 & 0.51 & 611 & 7442 & 0.0013 & & & & \\
\hline
\end{tabular}

The yield limit $\sigma_{Y}$ is taken as a function of $p$ and $\mathrm{d} \sigma_{Y} / \mathrm{d} p$ is the hardening modulus. The following nonlinear hardening law is adopted:

$$
\sigma_{Y}=\sigma_{0}+Q_{1}\left(1-\exp \left(-b_{1} p\right)\right)+Q_{2}\left(1-\exp \left(-b_{2} p\right)\right) .
$$

The first exponential function was used to represent the short nonlinear hardening regime after the initial yield stress. The second one, with a small value of $b_{2}$, can mimic the quasi-linear hardening regime prior to fracture (see Fig. 1). The parameters of the proposed constitutive equations were identified in the case of a nickel foam with relative density $\Phi^{\star}=0.035$. The experimental database contains the tensile tests along the directions RD and TD, including the information on lateral strain values. The calibrated parameters are given in Table 2. A tensile test along the direction RT at $45^{\circ}$ from RD and TD was also included in the identification procedure to identify $H_{d}$. Table 2 also gives some components of the orthotropic elasticity tensor obtained from tensile tests in direction TD, RD and RT, with unloading after a small amount of plastic strain. The simulated and experimental tensile curves are compared in Fig. 1. The plastic lateral contraction parameters obtained with this set of parameters are

$$
\text { tension along } \mathrm{RD}\left\{\begin{array} { l } 
{ v _ { 1 2 } ^ { p } = 0 . 4 9 , } \\
{ v _ { 1 3 } ^ { p } = 0 . 4 7 , }
\end{array} \quad \text { tension along } \mathrm { TD } \left\{\begin{array}{l}
v_{12}^{p}=0.17 \\
v_{13}^{p}=0.22
\end{array}\right.\right.
$$

These values are in good agreement with the experimental results of Table 1.

\subsection{Application to strain concentration around machined holes in a foam sheet}

After the identification procedure based on the overall tensile responses of nickel foam, the model can be applied to predict the strain field around a hole of given size embedded in a nickel foam plate. This was already done with success in the case of large holes $(R=12 \mathrm{~mm})$ in the reference (Badiche et al., 2000) where the finite element analysis is described. Fig. 5 shows quantitative comparisons of the axial strain field $\varepsilon_{22}$ as predicted by finite element analyses based on the anisotropic compressible plasticity model, and as measured from strain field measurements. The finite element analyses were carried out under plane stress conditions. A good agreement is found in the case of hole radii $R=4 \mathrm{~mm}$ and $R=2 \mathrm{~mm}$, as shown in Fig. 5(a)-(c) at different overall strain levels. Strain concentration factors at the equator ranging from 3 to 5 depending on the overall strain loading are predicted. The measured concentration factors are somewhat smaller.

This model, based on the classical continuum, predicts the same strain concentration factors around smaller holes. This contradicts the experimental results for $R=1 \mathrm{~mm}$ holes shown in Fig. 5(d). The strain heterogeneities detected around such holes are of the same order of magnitude as the fluctuations observed in nickel foam samples without any machined hole. The strain field measurement method reaches its limits since the strain values provided by the method are influenced by a distance of 3 cells (see the parameters defined in Section 2.2). If the strain concentration exists only over such a distance, it will not be detected with enough precision. As a matter of fact, it is found experimentally that fracture does not systematically start from such small machined holes. In two specimens out of three, the final crack leading to fracture initiated outside the hole region. Clearly the model based on classical compressible plasticity cannot account for this fact. 


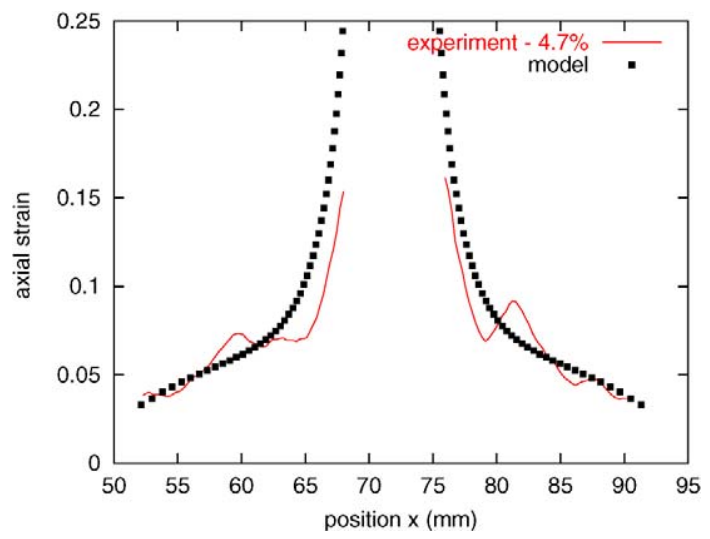

(a)

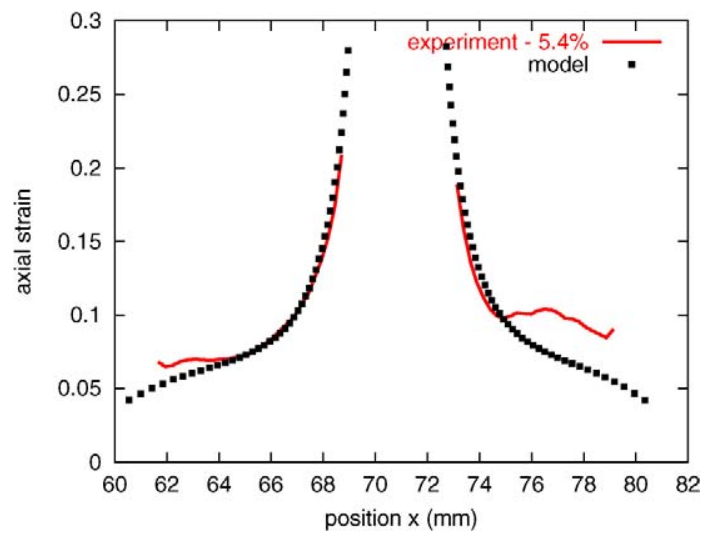

(c)

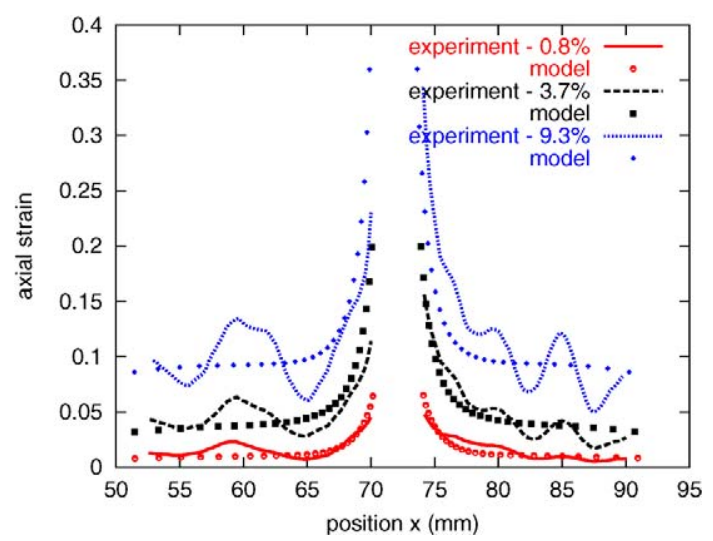

(b)

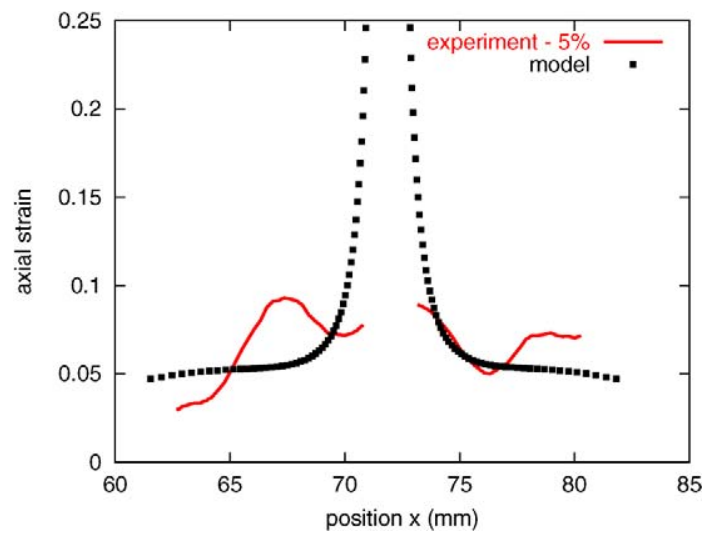

(d)

Fig. 5. Strain component $\varepsilon_{22}$ along the ligament of the nickel foam plate with a machined hole (coordinate axis $x_{1}$ ) from the strain field measurements at different overall strain levels $\varepsilon_{22}^{\infty}$ and three different hole radii. The experimental results from strain field measurements are compared to the finite element simulations. (a) $R=4 \mathrm{~mm}$ (specimen width $40 \mathrm{~mm}$ ); (b) $R=2 \mathrm{~mm}$ (specimen width $40 \mathrm{~mm}$ ); (c) $R=2 \mathrm{~mm}$ (specimen width $20 \mathrm{~mm}$ ); (d) $R=1 \mathrm{~mm}$ (specimen width $20 \mathrm{~mm}$ ). The tensile loading direction is TD.

\subsection{Presentation of the microfoam model}

The previous anisotropic compressible model can be incorporated into a micromorphic continuum framework involving only a limited number of additional parameters. The micromorphic continuum was introduced in Eringen and Suhubi (1964), Mindlin (1964). A complete presentation of this generalised continuum in the context of finite elastic-plastic deformations can be found in Eringen (1999), Forest and Sievert (2003). The kinematics and balance equations are recalled briefly within the small strain framework. The anisotropic compressible elastic-plastic constitutive equations are then proposed.

The motion of each material point of a micromorphic continuum is described by the displacement vector $\underline{\boldsymbol{u}}$ and by a full, nonsymmetric, microdeformation second order tensor $\chi$. The microdeformation accounts for the rotation and stretch of an underlying tryad of directors given at each material point. The microdeformation field is a priori independent from the gradient of the displacement field. It is generally not compatible. If only the skew-symmetric part of $\chi$ is taken into account in the theory, the micromorphic medium reduces to a Cosserat theory. The symmetric part of $\tilde{\chi}$ represents a microstrain. Both microstrain and microrotation are included in the present model. There are three strain measures: the strain tensor $\boldsymbol{\varepsilon}$, the relative deformation $\underset{\sim}{\boldsymbol{e}}$ and the microdeformation gradient $\boldsymbol{K}$,

$$
\underset{\sim}{\boldsymbol{\varepsilon}}=\frac{1}{2}\left(\nabla \underline{\boldsymbol{u}}+(\nabla \underline{\boldsymbol{u}})^{\mathrm{T}}\right), \quad \underset{\sim}{\boldsymbol{e}}=\nabla \underline{\boldsymbol{u}}-\underset{\sim}{\chi}, \quad \underset{\sim}{\boldsymbol{K}}=\nabla \underset{\sim}{\chi}
$$


The relative deformation measures the difference between the macro- and micro-deformation. The gradient of the microdeformation $\underset{\sim}{\boldsymbol{K}}$ is a third rank tensor. If an internal constraint forces $\underset{\sim}{\boldsymbol{e}}$ to vanish, the micromorphic theory reduces to a second gradient model (Mindlin and Eshel, 1968).

A stress tensor is associated to each strain measure in the power of internal forces:

$$
p^{(i)}=\underset{\sim}{\sigma}: \underset{\sim}{\dot{\varepsilon}}+\underset{\sim}{\boldsymbol{s}}: \underset{\sim}{\dot{\boldsymbol{e}}}+\underset{\sim}{\boldsymbol{S}}: \underset{\sim}{\dot{\boldsymbol{K}}}
$$

where $\underset{\sim}{\sigma}$ is the symmetric force stress tensor, $\underset{\sim}{\boldsymbol{s}}$ the generally nonsymmetric relative stress tensor and $\underset{\sim}{S}$ the third rank hyperstress tensor. The stress tensors must fulfil the following balance equations for momentum and moment of momentum:

$$
\nabla \cdot(\underset{\sim}{\sigma}+\underset{\sim}{s})=0, \quad \nabla \cdot \underset{\sim}{S}+\underset{\sim}{s}=0 .
$$

Volume forces, couples and double forces are not considered in the present work. The power of contact forces at the boundary or at any section of the body can be written as

$$
p^{(c)}=\underline{\boldsymbol{t}} \cdot \underline{\dot{\boldsymbol{u}}}+\underset{\sim}{\boldsymbol{M}}: \underset{\sim}{\dot{\chi}}
$$

where

$$
\underline{t}=(\underset{\sim}{\sigma}+\underline{s}) \cdot \underline{n}, \quad M=\underline{\sim} \cdot \underline{\boldsymbol{n}}
$$

respectively are the traction vector and the tensor of contact double forces.

In the proposed microfoam model, the classical strain tensor only is divided into elastic and plastic parts according to (2). The plastic flow is still described by Eqs. (3) to (7) where the symmetric stress tensor $\sigma$ only intervenes.

Simple linear relationships are assumed to link relative deformation and relative stress tensors, on the one hand, and the gradient of micro-deformation and the hyperstress tensor, on the other hand. The associated tensors of moduli are assumed to be isotropic for simplicity, contrary to the tensor of elastic moduli $\boldsymbol{C}$ which is still taken orthotropic in the simulations of the next sections. The representation of these constitutive tensors given in Mindlin (1964), Shu and Fleck (1999), Forest and Lorentz (2004) is adopted:

$$
\begin{aligned}
& \underset{\sim}{\boldsymbol{s}}=\lambda_{s}(\operatorname{trace} \underset{\sim}{\boldsymbol{e}}) \underset{\sim}{\mathbf{1}}+2 \mu_{s} \underset{\sim}{\boldsymbol{e}}{ }^{s}+2 \mu_{c s} \underset{\sim}{\boldsymbol{e}}, \\
& \underset{\sim}{\boldsymbol{S}}=l_{c}^{2}(\lambda \underset{\sim}{\mathbf{1}} \otimes \nabla(\operatorname{trace} \underset{\sim}{\boldsymbol{\chi}})+2 \mu \underset{\sim}{\boldsymbol{K}})
\end{aligned}
$$

where ${\underset{\sim}{\boldsymbol{e}}}^{s}$ and ${\underset{\boldsymbol{e}}{a}}^{a}$ respectively are the symmetric and skew-symmetric parts of the relative deformation $\underset{\sim}{\boldsymbol{e}}$. The moduli $\left(\lambda_{s}, \mu_{s}, \mu_{c s}\right)$ can be regarded as penalty factors that prevent the microdeformation from departing too much from the macrodeformation. For higher and higher values of $\left(\lambda_{s}, \mu_{s}, \mu_{c s}\right)$, the micromorphic model will be closer to a second gradient model. The moduli $(\lambda, \mu)$ are assumed to have the same order of magnitude as the classic elastic moduli of the foam. The main parameter remains then the characteristic length $l_{c}$.

Note that no direct physical meaning can be given to $l_{c}$ since the relevant quantity is the product $l_{c}^{2} \mu$ and $l_{c}^{2} \lambda$ in the model. However the characteristic sizes of the plastic deformation zones in boundary layers or around holes are related to $l_{c}$. Physically relevant characteristic lengths arise in fact by solving boundary value problems. A different characteristic length can be found for each type of problem, even though they can be expected to keep the same order of magnitude for a simple model like microfoam. An example of derivation of a characteristic length in a problem of inhomogeneous tension/compression is given in Appendix A. The found characteristic length is proportional to $l_{c}$, the factor being related to the hardening modulus $\mathrm{d} \sigma_{Y} / \mathrm{d} p$ and the standard elastic moduli.

This simplified version of micromorphic elastoplasticity was shown in Forest and Lorentz (2004), Forest et al. (2005) to be sufficient to regularise the simulation of strain localisation bands in isotropic aluminium foams under compression. More general elastoplastic micromorphic models for incompressible materials and compressible geomaterials can be found in Forest and Sievert (2003), Chambon et al. (2001) respectively. The microfoam model is shown in the next section to be sufficient to describe some aspects of the deformation and fracture of nickel foams. 


\section{Continuum modelling of the hole size effect in nickel foams}

The previous anisotropic compressible plasticity model was implemented in a finite element program based on a standard variational formulation of the balance equations (11). In the two-dimensional case, there are six independent degrees of freedom

$$
[\mathrm{DOF}]=\left[\begin{array}{llllll}
u_{1} & u_{2} & \chi_{11} & \chi_{22} & \chi_{12} & \chi_{21}
\end{array}\right] .
$$

The reader is referred to the reference (Forest and Lorentz, 2004) for the details of the finite element implementation. The nonlinear constitutive equations are integrated using a Runge-Kutta method involving second order derivatives. Quadratic elements with reduced integration are used for all simulations of this work.

\subsection{Finite element analysis of the problem of a hole in an elastoplastic micromorphic medium}

The analysis of stress and strain concentrations at holes, edges, cracks or other singularities were a strong incentive for the development of the mechanics of generalised continua in the early 1960s. The aim was to investigate the effect of the introduction of an intrinsic length in continuum theory, on stress-strain enhancement at holes or cracks. In the case of linear elasticity, Mindlin analysed the stress concentration factor at a hole in a plate for a couple-stress medium (Mindlin, 1962). Contrary to the classical situation, the stress concentration factor was found to depend on the relative size of the hole with respect to the value of the characteristic length, even if the hole is embedded in an infinite matrix. The analytical solution of the more general problem of the spherical or cylindrical elastic inclusion inside an infinite matrix was solved only recently for linear Cosserat elasticity (Cheng and He, 1995, 1997; Zhang and Sharma, 2005). Finite element simulations within the Cosserat framework show that, contrary to the classical situation, the stress-strain state is generally not homogeneous inside a spherical or cylindrical elastic heterogeneity (Forest et al., 1999). The stress concentration factor at the equator of a spherical hole in an infinite linear elastic Cosserat matrix tends asymptotically to the classical constant value for large enough holes. For holes with a radius close to or smaller than the value of the intrinsic lengths of the Cosserat matrix, the factor is found to decrease. The value for vanishingly small holes tends toward an asymptotic limit that depends on the Cosserat intrinsic length scale and on the additional Cosserat couple modulus $\mu_{c} \geqslant 0$. It remains larger than one (Forest, 2004), meaning that holes of any size induce stress concentration in a traditional Cosserat medium.

The strain field around a hard particle in a plastic second gradient continuum matrix was studied via the finite element method in Xue et al. (2000). The case of a void in a single crystal matrix regarded as a second gradient medium was tackled in Shu (1998). From the literature, however, it is not clear whether stress-strain concentrations are reduced or not at a vanishingly small hole in a second grade medium. In Neff and Forest (2005), finite element analyses were provided showing that the strain concentration factor at a hole in a linear elastic micromorphic continuum decreases to one when the hole size tends to zero. In the present section, this question is addressed again in the case of the microfoam model.

The strain field $\varepsilon_{22}$ around holes in quasi-infinite nickel foams plates is presented in Fig. 6 as predicted by finite element computations with the microfoam model. The material parameters specifically used for these simulations are given in Table 3. Their identification is discussed in the next subsection. The results are provided for different hole sizes ranging from $R=0.5 \mathrm{~mm}$ to $R=4 \mathrm{~mm}$. The results obtained using the classical formulation of the anisotropic compressible plasticity model is recalled in Fig. 6(a) as a reference. The finite element computations were carried out under the following plane stress conditions

$$
\begin{aligned}
& \sigma_{33}=s_{33}=0, \\
& S_{i j 3}=0 .
\end{aligned}
$$

Table 3

Specific parameters of the microfoam model used for the simulation of the hole size effect in nickel foams

\begin{tabular}{llllll}
\hline$l_{c}$ & $\lambda$ & $\mu$ & $\lambda_{s}$ & $\mu_{s}$ & $\mu_{c s}$ \\
$(\mathrm{~mm})$ & $(\mathrm{MPa})$ & $(\mathrm{MPa})$ & $(\mathrm{MPa})$ & $(\mathrm{MPa})$ & $(\mathrm{MPa})$ \\
\hline 0.1 & 111 & 167 & 11000 & 17000 & 10000 \\
\hline
\end{tabular}




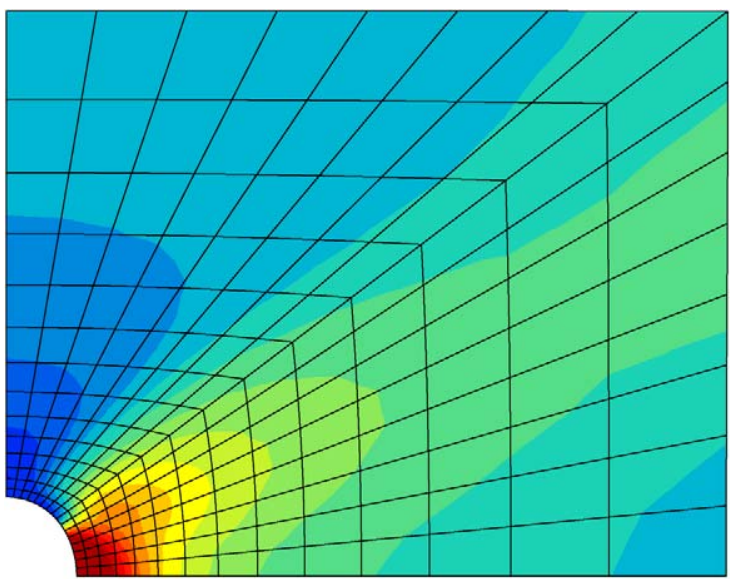

(a)

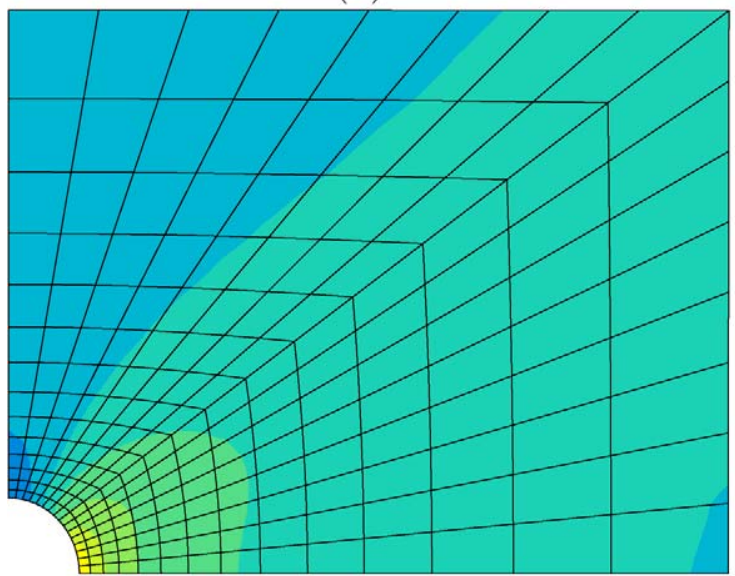

(c)

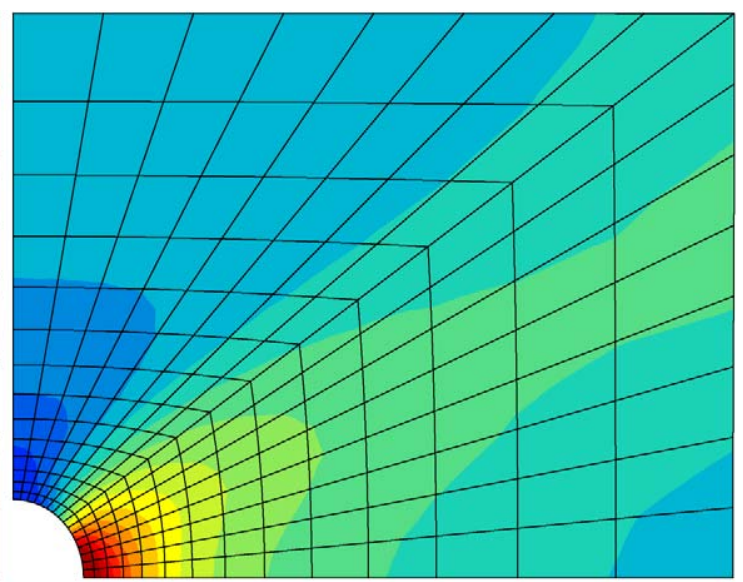

(b)

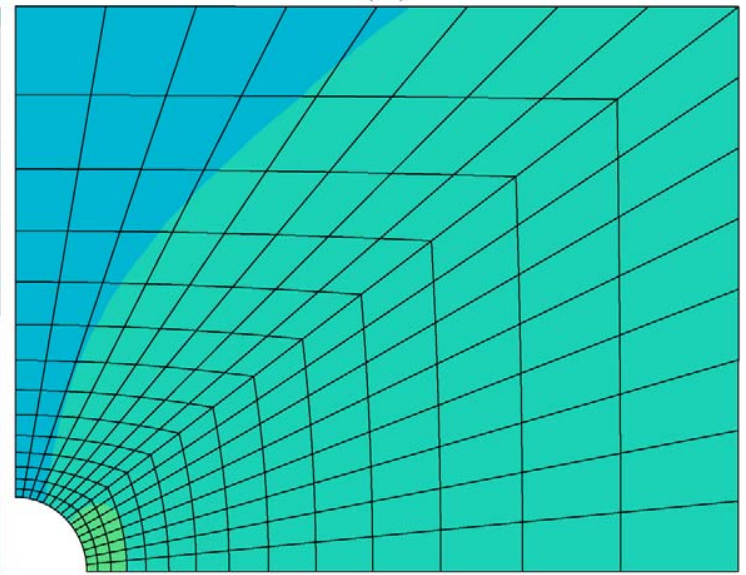

(d)

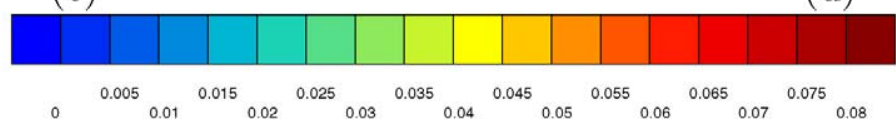

Fig. 6. Finite element simulations of the strain field $\varepsilon_{22}$ around a hole in a nickel foam plate in tension along the vertical axis 2 : (a) simulation based on the classical continuum; (b) microfoam model for $R=4 \mathrm{~mm}$; (c) microfoam model for $R=1 \mathrm{~mm}$; (d) microfoam model for $R=0.5 \mathrm{~mm}$. The overall strain level prescribed at infinity is $\varepsilon_{22}^{\infty}=0.02$. The tensile loading direction is TD.

One quarter only of the finite element mesh is considered in the simulation and shown on Fig. 6 . The symmetries of the solution give information on the boundary conditions to be applied on the ligament (horizontal line going through the equator) and on the vertical line going through the pole. The conditions on the displacement and some conditions on the microdeformation are clear: $U_{2}=0, \chi_{12}=\chi_{21}=0$ (on the ligament) and $U_{1}=0, \chi_{12}=\chi_{21}=0$ (vertical symmetry axis) respectively. But no information on $\chi_{11}$ and $\chi_{22}$ can be deduced from the symmetry conditions. Conditions about the associated forces $M_{i j}$ can be derived from the even character of the functions $\chi_{11}$ and $\chi_{22}$. Their gradient with respect to $x_{1}$ vanish on the horizontal ligament. So do $M_{11}$ and $M_{22}$ because of the linear relationship between $\underline{S}$ and $\boldsymbol{K}$.

The most striking result is that the strain field $\varepsilon_{22}$ around the hole becomes smoother as the hole size decreases. For large holes $R>10 \mathrm{~mm}$, the strain field in the micromorphic continuum cannot be distinguished from the classical result. For $R=4 \mathrm{~mm}$ (Fig. 6(b)), the strain field remains very close to the classical result of Fig. 6(a). For $R=1 \mathrm{~mm}$ (Fig. 6(c)), the strain concentration is considerably reduced at the equator when compared to the reference situation. For $R=0.5 \mathrm{~mm}$ (Fig. 6(d)), the strain concentration almost disappears. More quantitative results are presented and discussed in the next subsection. 


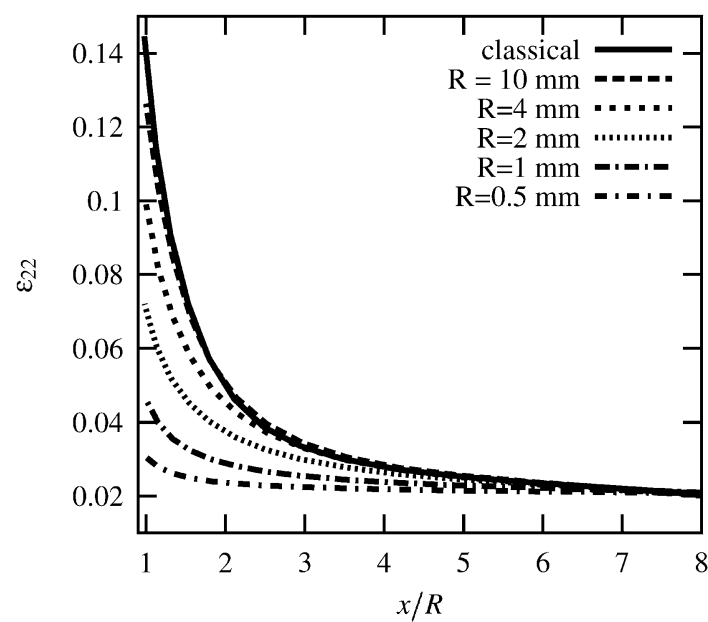

Fig. 7. Strain component $\varepsilon_{22}$ along the ligament of the nickel foam plate with a machined hole (coordinate axis $x_{1}$ ) from finite element simulations with the microfoam model for different hole radii. The overall strain level prescribed at infinity is $\varepsilon_{22}^{\infty}=0.02$. The tensile loading direction is TD.

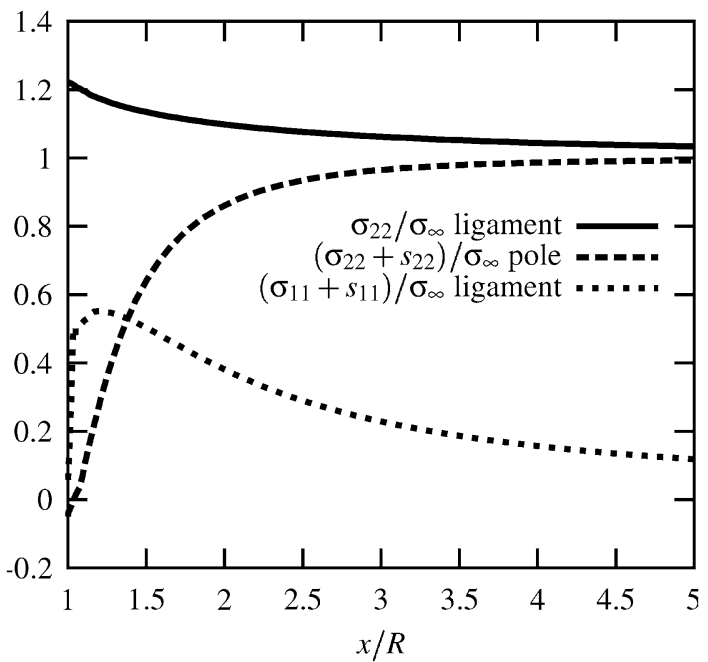

Fig. 8. Stress profiles along the ligament and along the vertical line going through the pole. The overall strain level prescribed at infinity is $\varepsilon_{22}^{\infty}=0.02$. The tensile loading direction is TD.

\subsection{Identification of the characteristic length}

The idea of identifying the characteristic length of a generalised continuum model from strain field measurements was put forward and illustrated in the references (Geers et al., 1998, 1999). The strain field was measured around the crack tip in a CT specimen made of a glass-fiber reinforced polymer composite. In the present work, the intrinsic length $l_{c}$ was estimated from the strain field measurement around holes in nickel foams. The identified length $l_{c}=0.1 \mathrm{~mm}$ is such that the strain concentration factor decreases for hole sizes smaller than $R=4 \mathrm{~mm}$. The strain component $\varepsilon_{22}$ is plotted along the ligament in Fig. 7 for hole sizes ranging from $10 \mathrm{~mm}$ to $0.5 \mathrm{~mm}$. It is found that this component is almost homogeneous along the ligament for $R=1 \mathrm{~mm}$ and $R=0.5 \mathrm{~mm}$, as it should be, since a hole can then hardly be distinguished from a large cell. For $R=10 \mathrm{~mm}$, the profile is almost identical to the reference distribution. For $R=4 \mathrm{~mm}$, the strain concentration already decreases but remains significant as observed experimentally.

In the identification procedure, the parameters $\lambda, \mu, \lambda_{s}, \mu_{s}, \mu_{c s}$ of Table 3 were fixed once for all and $l_{c}$ was varied. The values $\lambda, \mu$ are constants close to the classical elastic moduli of the foams and $\lambda_{s}, \mu_{s}, \mu_{c s}$ are treated as penalty factors that force the microdeformation to remain close to the gradient of the displacement field (strain gradient approximation).

A deeper understanding of the model can be gained by looking at the computed stress components. The stress concentration $\sigma_{22} / \sigma_{\infty}\left(\sigma_{\infty}\right.$ being the stress value of $\sigma_{22}$ far from the hole) is plotted along the ligament for a hole $R=1 \mathrm{~mm}$ after $\varepsilon_{22}^{\infty}=0.02$ of overall deformation, in Fig. 8. It takes the value 1.2 at the equator. The evolution of the $\sigma_{11}+s_{11}$ along the ligament is also given to check that it tends to 0 , in a progressive way far from the hole and quite abruptly at the equator. The boundary condition $t_{1}=0$ at $x=R$ implies in fact that $\sigma_{11}+s_{11}$ should indeed vanish (see Eq. (13) with $\underline{\boldsymbol{n}}=-\underline{\boldsymbol{e}}_{1}$ ). For similar reasons, it is expected that $\sigma_{22}+s_{22}$ should vanish at the pole of the hole since there $t_{2}=0$. It is indeed the case as shown by the profile of $\sigma_{22}+s_{22}$ along the vertical line going through the pole, shown in Fig. 8.

\section{Deformation of central crack panels}

The objective of this section is to show that the microfoam model can be used to predict the initiation of crack growth in nickel foams. For that purpose a simple ductile fracture criterion is incorporated into the constitutive framework. 
Table 4

Fracture stress and strains measured in the tension of foam strips along three material directions

\begin{tabular}{lll}
\hline Tensile direction & Fracture stress $(\mathrm{MPa})$ & Fracture strain \\
\hline $\mathrm{RD}$ & $1.65 \pm 0.05$ & $0.097 \pm 0.019$ \\
$45^{\circ}$ & $1.33 \pm 0.09$ & $0.121 \pm 0.014$ \\
$\mathrm{TD}$ & $1.10 \pm 0.1$ & $0.173 \pm 0.019$ \\
\hline
\end{tabular}

\subsection{A simple anisotropic ductile fracture model}

The ductile fracture mechanisms of nickel foams were studied by SEM microscopy and micro-tomography from tensile tests on initially sane or pre-cracked foam samples in Badiche et al. (2000), Dillard (2004), Dillard et al. (2005). They involve complex tearing mainly at the nodes of the foam microstructure and the existence of a diffuse damage zone around macroscopic cracks. The local fracture phenomena combine intragranular and intergranular cracking of the nickel grains. The size of the damage zone, i.e. the distance from the main crack tip at which damaged struts and nodes are still visible, was estimated to be about 4 to 5 cells from 3D tomographic images.

Detailed ductile fracture models are far less developed for metal foams than for dense metals (Besson, 2004). We propose here a simple model accounting for the fracture stress and strain of the foam only.

The fracture stress and strain were recorded in a series of tensile tests on nickel foam strips (specimen width: $20 \mathrm{~mm}$ ). The tests were carried out in direction RD, TD and at $45^{\circ}$. Ten tests were performed for each direction to investigate the possible scatter in fracture stress and strain. The mean values and standard deviations measured are recorded in Table 4. The fracture stress is defined here as the maximum stress value in the tensile curve. The fracture strain is defined here as the strain value for which this maximum stress is reached. The tensile fracture behaviour of nickel foams turns out to be strongly anisotropic. The fracture strain is about 0.09 for tension along RD and reaches 0.17 for tension along TD. The observed scatter in the fracture strain can be neglected in a first analysis compared to the anisotropy effect.

A fracture criterion based on the cumulative plastic strain $p$ is proposed. The cumulative plastic strain is obtained by integrating the plastic multiplier introduced in Eqs. (5) and (6). It fulfils the following requirement:

$$
\underset{\sim}{\sigma}:{\underset{\sim}{\dot{\varepsilon}}}^{\mathrm{p}}=\sigma_{\mathrm{eq}} \dot{p}
$$

where the equivalent stress measure was defined in (3). It can also be computed as a function of the plastic strain rate tensor. For that purpose, the plastic flow rule (5) can be rewritten as

$$
\dot{\sim}^{\mathrm{p}}=\dot{p} \underset{\underset{\sim}{\boldsymbol{L}}: \underset{\sim}{\boldsymbol{\sigma}}}{\sigma_{\mathrm{eq}}} .
$$

In the coordinate system associated with the material anisotropy axes $(R D, T D, N D)$, the components of the matrix $[\underset{\sim}{\boldsymbol{L}}] \mathrm{read}$

$$
[\underset{\sim}{\boldsymbol{L}}]=\left[\begin{array}{cccccc}
\frac{C}{6}\left(4 H_{a}+H_{b}+H_{c}\right)+F P^{2} & -\frac{C}{6}\left(2 H_{a}+2 H_{b}-H_{c}\right)+F P Q & -\frac{C}{6}\left(2 H_{a}-H_{b}+2 H_{c}\right)+F P R & 0 & 0 & 0 \\
-\frac{C}{6}\left(2 H_{a}+2 H_{b}-H_{c}\right)+F P Q & \frac{C}{6}\left(H_{a}+4 H_{b}+H_{c}\right)+F Q^{2} & -\frac{C}{6}\left(-H_{a}+2 H_{b}+2 H_{c}\right)+F Q R & 0 & 0 & 0 \\
-\frac{C}{6}\left(2 H_{a}-H_{b}+2 H_{c}\right)+F P R & -\frac{C}{6}\left(-H_{a}+2 H_{b}+2 H_{c}\right)+F Q R & \frac{C}{6}\left(H_{a}+H_{b}+4 H_{c}\right)+F R^{2} & 0 & 0 & 0 \\
0 & 0 & 0 & \frac{3}{2} C H_{d} & 0 & 0 \\
0 & 0 & 0 & 0 & \frac{3}{2} C H_{e} & 0 \\
0 & 0 & 0 & 0 & 0 & \frac{3}{2} C H_{f}
\end{array}\right] .
$$

This matrix is generally invertible (at least for the parameters considered in this work) so that the plastic multiplier can be deduced from the previous equation as

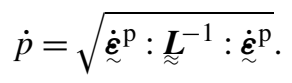

It can then be regarded as an anisotropic norm of the plastic strain rate tensor.

When the equivalent plastic strain $p$ reaches a critical value $p_{c}$ at a material point in the simulation, an explicit softening term is added to the function $\sigma_{Y}(p)$ defined by (7). The softening function contains a parabolic and an exponential term that depend on the variable $p$ only. The final limit stress can be chosen as close to zero as necessary. 


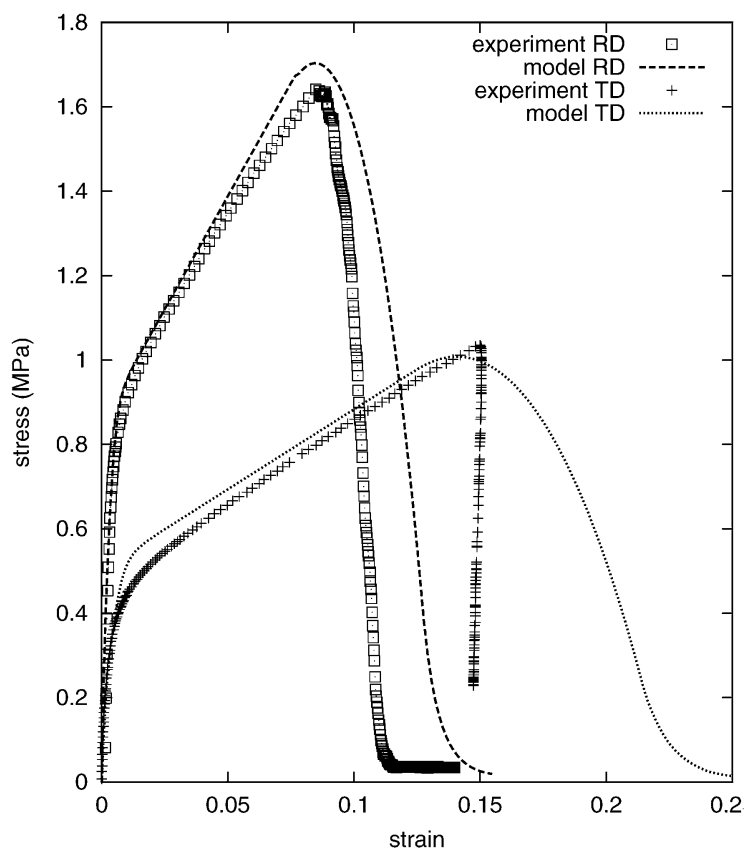

Fig. 9. Tensile tests in the direction RD and TD conducted until fracture on foam strips: experimental and simulated results. In the presented tension test in direction TD, the final crack initiated outside the gauge length.

Plastic strain occurring at $p>p_{c}$ is not due only to the plastic deformation of nickel struts but is a phenomenological measure of irreversible deformation associated with local damage and fracture. The simulated tensile curves are given in Fig. 9. They show the almost linear hardening regime and the constitutive softening branch corresponding to the damage and fracture of the material point. The simulated softening part of the tensile curve in direction RD is more ductile than in the experiment (see Fig. 9). The model should be improved in this softening part. However, in this simple version of the model, the aim remains the prediction of the maximum stress for the different loading directions. The anisotropic character of the fracture criterion clearly appears since the fracture strain for tension along TD is almost twice the corresponding value for tension along RD. The critical equivalent plastic strain $p_{c}$ was calibrated with respect to the fracture strain observed in tension along RD. The value $p_{c}=0.085$ was chosen. The simulated fracture strain for a tensile test in the direction TD is therefore a prediction of the model. In the model, the anisotropy of fracture turns out to be driven by the anisotropy of the plastic behaviour since the anisotropy matrix $\underset{\sim}{\boldsymbol{L}}$ entering Eq. (18) is the same as the matrix accounting for the anisotropic tensile behaviour. It is based on the coefficients of Table 2. This seems to be a simple but realistic assumption since there is a quite good agreement between the predicted and experimental values of the maximum stress and the corresponding strain for tension along TD, as shown on Fig. 9.

Once a fracture criterion is chosen at the level of the material point, it must be tested at the structural level. The model involves a softening regime for $p>p_{c}$. As a result strain localisation phenomena are expected to occur in structural computations.

\subsection{Finite element simulations of strain localisation at a crack tip in an elastoplastic micromorphic medium}

The constitutive and fracture model is now applied to predict the response of a $56 \mathrm{~mm}$-wide nickel foam panel with a $10 \mathrm{~mm}$-long central crack. One first considers the case of a crack parallel to the direction TD for tensile loading of the plate along the direction RD. The finite element simulation is carried out first using the classical continuum and the plasticity/damage model identified in the previous section. Plane stress conditions are enforced in the $2 \mathrm{D}$ simulation. Plastic strain develops first in the near crack tip region and, at some loading instant, the critical value $p=p_{c}$ is reached locally. The subsequent local softening regime leads to strain localisation close to the crack tip. This localisation zone then extends in direction TD, which mimics crack propagation. The overall curve giving the resulting force divided by the ligament length as a function of normalised displacement is shown in Fig. 10 (a total gauge length of $70 \mathrm{~mm}$ is 


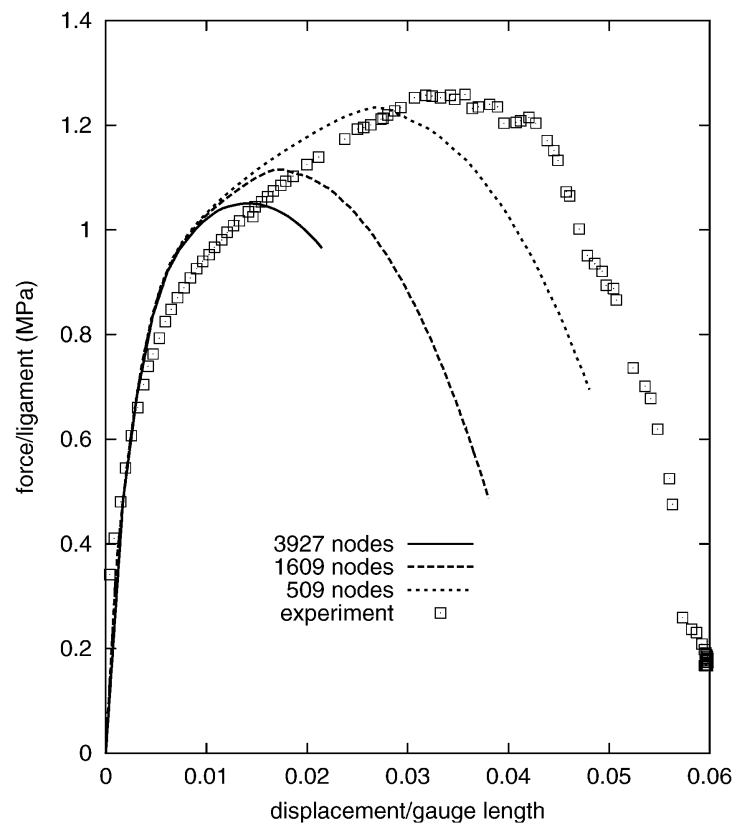

Fig. 10. Force/displacement curves of a nickel foam plate with a central crack under mode I loading: experimental results and finite element simulations based on the classical elastoplastic continuum for three different mesh sizes. The crack and the loading axis were respectively parallel to the TD and RD directions.

taken here). The force first increases and decreases after a certain loading instant. The decreasing branch of the curve coincides with crack propagation. The simulations were carried out for different mesh sizes. As expected, a strong mesh dependence was found as shown in Fig. 10. A finer mesh systematically leads to an earlier initiation of crack growth. Accordingly, it is not possible to predict the initiation of crack growth based on these simulations. This is a known feature of the simulation of strain localisation and damage using softening models in a classical continuum and with classical finite elements (Borst et al., 1993).

The regularisation power of the microfoam model is now investigated by introducing the previous fracture model into the micromorphic framework. For that purpose, we simply use the same softening branch in the function $\sigma_{Y}(p)$ in the microfoam model as in the previous classical approach. This is possible because the anisotropic compressible plasticity criterion and flow rules are the same in the classical and micromorphic frameworks. The new ingredient is related to the characteristic length $l_{c}$ which enters Eq. (15). The simulations of the tensile response of a central crack panel made of nickel foam are performed again for different mesh sizes, in the case of the micromorphic continuum. The results are shown in Fig. 11 only for one quarter of the mesh and for four different meshes. The size of the strain localisation zone is shown to be almost mesh independent. This leads to a quasi mesh-independent overall loading curve (see Fig. 12) provided that sufficiently fine meshes are used. The initiation of crack growth can then be defined unambiguously. It is compared to experimental results in the next subsection.

Although the numerical results suggest a proper regularisation of the localisation problem by the microfoam model, we do not claim that it is the case, since the proof for that requires another study. Another hint of the regularising power of the micromorphic model is given in Appendix B, where a comparison is drawn between the present model and the so-called implicit gradient model proposed in Peerlings et al. (2001). The appendix shows that, in a simplified case, a Helmholtz equation for the microstrain can be derived from the balance of generalised moment of momentum.

\subsection{Comparison with experimental results}

Two views of a central crack panel made of nickel foam are shown in Fig. 13 before and after crack extension. The tensile axis is vertical and the crack is horizontal. Crack propagation is dominated by the remote mode I loading conditions, as a result of which the crack path remains approximately horizontal. A specific feature of cracking in ductile metal foams is the extended damage zone around the crack and the existence of some struts bridging the 

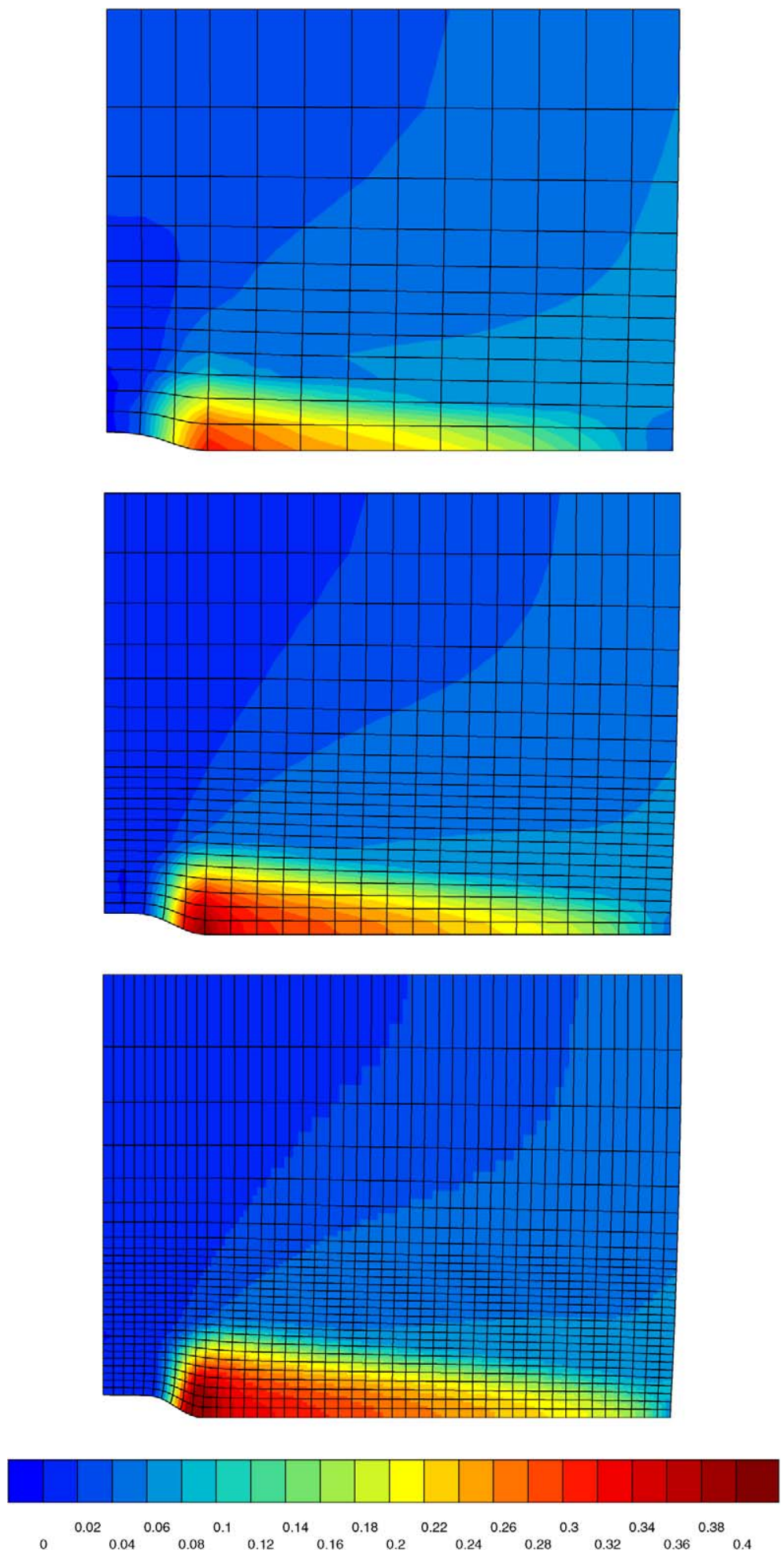

Fig. 11. Maps of equivalent plastic strain $p$ around the crack tip from finite element simulations based on the microfoam model for three different meshes (509, 1609 and 3927 visible nodes). Only one quarter of the mesh is shown. The vertical loading direction is RD. The maps are given for a value of displacement/gauge length equal to 0.058 (see the global curve of Fig. 12). 


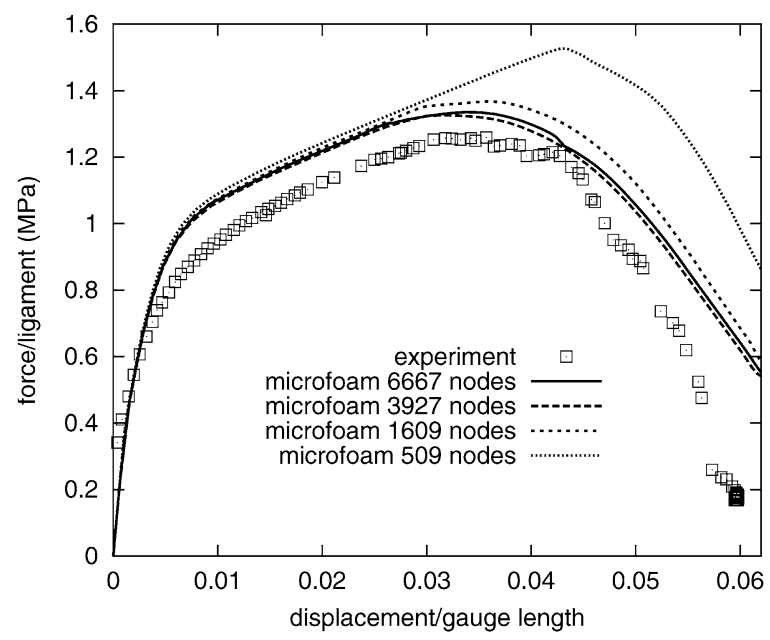

Fig. 12. Load/displacement curves of the tension of a nickel foam plate with a central crack: experimental results and finite element simulations based on the microfoam continuum for four different meshes. The loading direction is $\mathrm{RD}\left(l_{c}=0.06 \mathrm{~mm}\right)$.

crack behind the crack tip. Individual teared struts are visible in Fig. 13(b). The extension of the damage zone, i.e. the maximum distance from the crack tip at which damage (cracked) struts are observed, was estimated from microtomography analyses in Dillard et al. (2005). The distance was estimated to that of about 4 to 5 cells, i.e. about $2 \mathrm{~mm}$.

The micromorphic continuum model must be able to reproduce both the overall loading curve and the damage zone size. In the simulation the damage zone can be estimated by looking at the equivalent plastic strain maps $p$ as in Fig. 11. Both features of crack extension are related to the characteristic length $l_{c}$ incorporated in the microfoam model. The crack extension process was first simulated for the value $l_{c}=0.1 \mathrm{~mm}$ identified in Section 4.2 from the strain field around holes. Fig. 14 shows that this value leads to an overestimation of the maximum load and initiation of crack extension. A smaller value $l_{c}=0.06 \mathrm{~mm}$ is needed to correctly describe the overall force/displacement curve of a cracked panel in tension along the RD direction. It confirms that a length scale related to size effects generally is different from a length scale related to localisation. This may be a reason why the model, which contains only one length scale parameter, accounts only approximately for both effects at the same time. The tensile response of the foam is still slightly overestimated from the very beginning of the plastic regime, but it was also the case with the classical model (see Fig. 10) and the difference remains within the scatter observed on tensile curves in nickel foams. The equivalent plastic strain fields of Fig. 11 were obtained with this value of the characteristic length. The predicted extension of the high plastic deformation zone is also in reasonable agreement with the experimental observations, namely 4 to 5 cells.

A tension test on a cracked panel along the direction TD was then simulated to check the predictive capability of the microfoam model. The crack is aligned with direction RD and loaded under mode I conditions. The comparison between the experimental and simulation results is given in Fig. 15. The anisotropy of the plastic behaviour of the foam leads to a delayed propagation of the crack, compared to the case of a cracked panel loaded in direction RD. The relative displacement level at which the crack extends is almost twice larger for tension in direction TD than for direction RD. The microfoam model correctly accounts for this anisotropic fracture behaviour even though it slightly overestimates the maximum load and inception of crack extension.

\section{Conclusions}

An anisotropic compressible plasticity model was extended to incorporate intrinsic length scale effects within the framework of a micromorphic continuum. This framework was simplified in such a way that only one characteristic length had to be identified in addition to the material parameters of the classical plasticity model. Finite element simulations with this generalised continuum model, called microfoam, have revealed a remarkable feature of the nonlinear micromorphic continuum, namely the fact that strain concentrations around a hole in an plate are reduced and tend to disappear when the size of the hole is decreased. This new property of this continuum does not seem to 


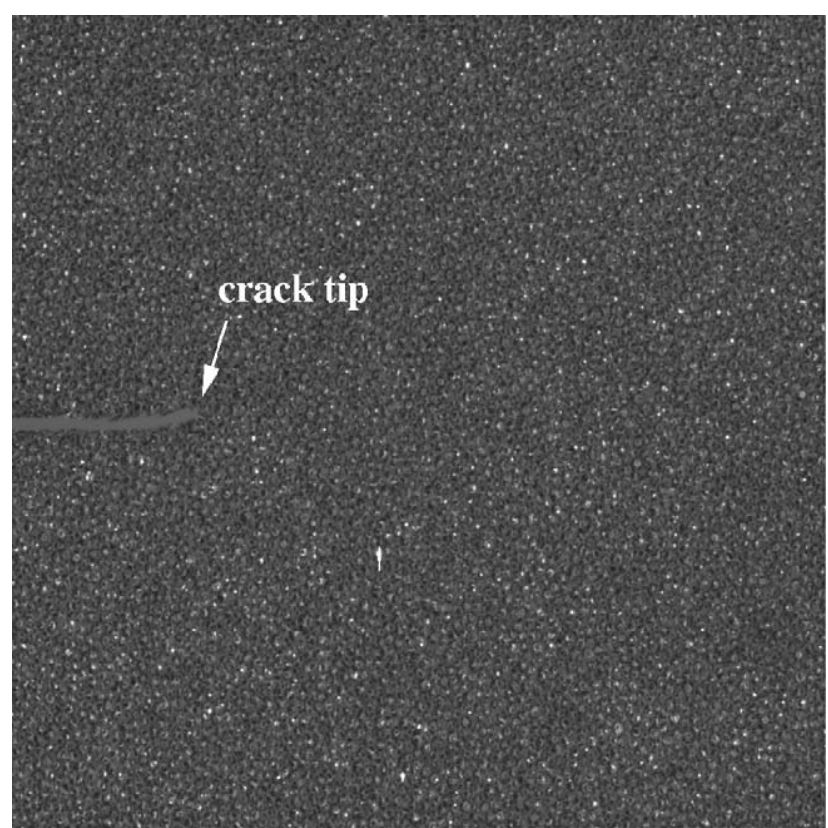

(a)

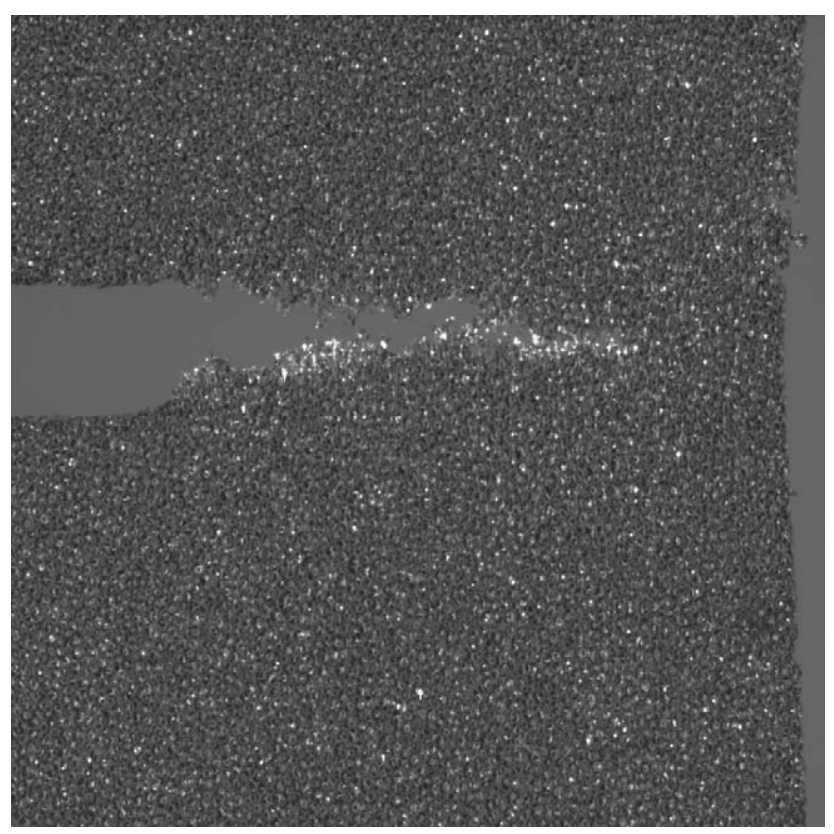

(b)

Fig. 13. (a) Central crack in a nickel foam plate. Only two thirds of the initial crack length are shown. The initial total crack length is $10 \mathrm{~mm}$. (b) Crack propagation under mode I conditions. The loading axis is vertical and coincides with direction RD.

have been noticed in literature. It was used to model the plastic behaviour of nickel foam plates with a hole, for which the strain concentration effect vanishes when the hole size becomes comparable to the cell size.

Experimental tests on nickel foam plates with a machined hole have shown that the final crack leading to fracture does not systematically initiate at the hole when the hole radius is equal to $1 \mathrm{~mm}$ (to be compared with the cell size $0.5 \mathrm{~mm}$ ). For holes with radii larger or equal to $2 \mathrm{~mm}$, the classical anisotropic compressible plasticity model was able to describe accurately the strain field around the hole as measured by a strain field measurement method based on image correlation. The intrinsic length scale $l_{c}$ of the microfoam model could be calibrated in such a way that the 


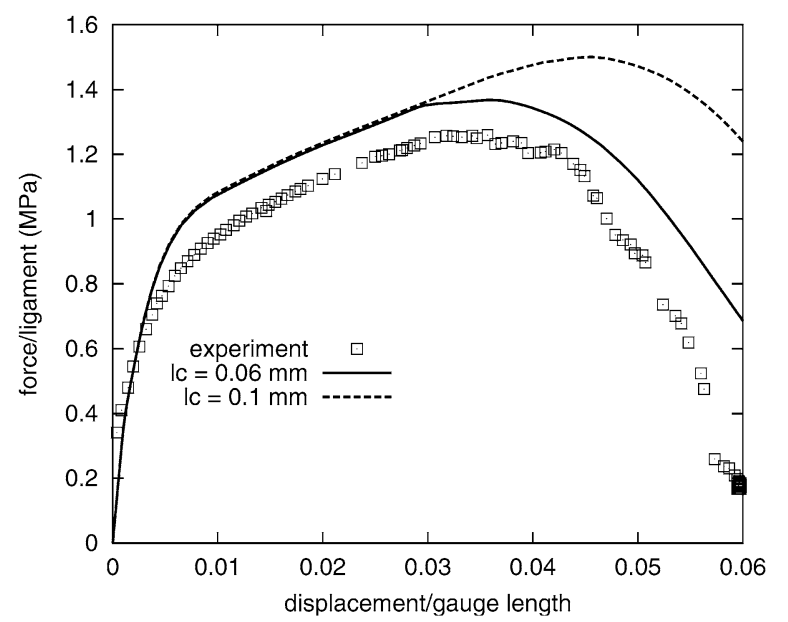

Fig. 14. Load/displacement curves of the tension of a nickel foam plate with a central crack: experimental results and finite element simulations based on the microfoam continuum for two different values of the intrinsic length $l_{c}$. The loading direction is RD.

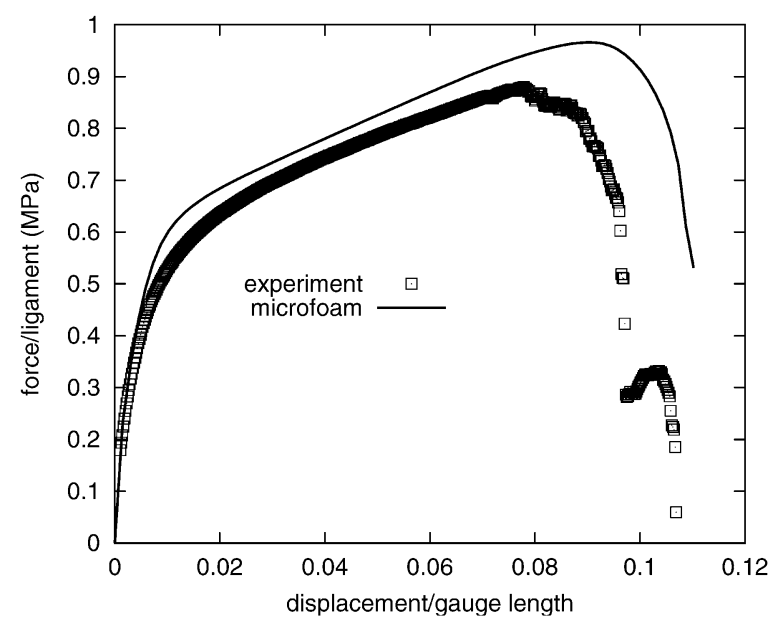

Fig. 15. Prediction of the microfoam model of the tensile behaviour of a central crack panel with a crack parallel to $\mathrm{RD}$ and comparison with the experimental result. The loading axis is $\operatorname{TD}\left(l_{c}=0.06 \mathrm{~mm}\right)$.

strain concentration around the hole is considerably reduced around a hole of radius $R=1 \mathrm{~mm}$. In general, it cannot be expected that a continuum model gives at the scale of 2 to 4 cells a correct description of the actual discrete behaviour of the cells. However, we have shown that the asymptotic behaviour of the proposed model for smaller and smaller holes is clearly defined and is in accordance with the intuitive and experimental view that small holes machined in a foam should no induce significant stress concentrations. An important consequence of this fact is that structures with complex geometries can be computed using this model even if they contain small geometrical details with a size of the order of or below 4 cells. These geometrical singularities will not induce spurious stress concentrations that would lead to earlier failure of the component.

The case of a central crack in a quasi-infinite nickel foam plate was then investigated based on an extension of the microfoam model. For that purpose, a softening branch was introduced in the phenomenological hardening function in order to mimic the deformation and fracture of tensile specimens in directions RD and TD. The provided finite element simulations have shown that the model was able to predict the initiation of crack propagation simulated as strain localisation at the crack tip. The critical parameter is an anisotropic measure of equivalent plastic strain which seems to be sufficient to explain the strongly anisotropic response of cracks oriented along the RD or TD directions under mode I loading conditions. Both the experimental overall load/displacement curves and typical size of the plasticity/damage zone could be reproduced in the simulation. The application of the strain field measurement method remains to be applied to the crack tip deformation field in the nickel foams for quantitative comparisons with the finite element simulations. Such measurements are available for open-cell aluminium foams in Andrews and Gibson (2002).

One single characteristic length was not sufficient to account for both the hole size effect and the initiation of crack propagation. Two characteristic lengths were determined: one characterising the plastic behaviour of the foam, one characterising some fracture properties. They have the same order of magnitude. A complete model should therefore incorporate both length scales or an evolving length scale, which was not the case in the present simple formulation of the microfoam model. In the general constitutive framework proposed for nonlinear micromorphic media in Forest and Sievert (2003), an intrinsic characteristic length is attached to each nonlinear mechanism which is accounted for. Several competing length scales can be introduced simultaneously. Alternative strain gradient plasticity continuum models based on multiple or evolving length scales as proposed by Fleck and Hutchinson (2001), Voyiadjis and Abu Al-Rub (2005), could also be used for that purpose. The attention will be focused in future work on the links between the newly introduced macroscopic degrees of freedom $\chi$ and the actual deformation of individual cells in the nickel foam since microtomographic analyses have made such detailed microstructure information available (Dillard et al., 2005). 


\section{Appendix A. Characteristic length of the microfoam model in a simple one-dimensional situation}

A micromorphic compressible elastoplastic material is considered, that admits a simple linear hardening rule:

$$
\sigma_{Y}=\sigma_{0}+H p
$$

and the elliptic yield function (3). The isotropic case $\underset{\widetilde{\sim}}{\boldsymbol{H}}=\underset{\widetilde{\sim}}{\mathbf{1}}$ and $\underset{\sim}{\boldsymbol{P}}=\underset{\sim}{\mathbf{1}}$, and also for elasticity, is considered. We choose $F=C / 2$ (and also a vanishing Poisson ratio to simplify the analytical derivation). This value ensures that no lateral deformation takes place during uniaxial loading. The problem becomes actually one-dimensional so that an analytical solution can be worked out for tension along direction 2:

$$
\sigma_{\text {eq }}=\sqrt{\frac{3 C}{2}} \sigma_{22}, \quad \dot{\varepsilon}_{22}^{p}=\dot{p} \sqrt{\frac{3 C}{2}}, \quad \dot{p}=\frac{2 \mu \sqrt{3 C / 2}}{3 \mu C+H} \dot{\varepsilon}_{22}
$$

where $\mu$ is the shear modulus. For monotonous loading, in the plastic regime, one gets:

$$
p=\frac{2 \mu \sqrt{3 C / 2}}{3 \mu C+H}\left(\varepsilon_{22}-\frac{\sigma_{0}}{2 \mu \sqrt{3 C / 2}}\right) .
$$

The balance equations (11) for stresses and the elasticity relations (15) must be taken into account:

$$
\begin{aligned}
& \left(\sigma_{22}+s_{22}\right)_{, 2}=0, \quad S_{222,2}+s_{22}=0, \\
& \sigma_{22}=2 \mu\left(\varepsilon_{22}-\varepsilon_{22}^{p}\right)=\frac{2 \mu}{3 \mu C+H}\left(H \varepsilon_{22}+\sigma_{0} \sqrt{\frac{3 C}{2}}\right), \\
& s_{22}=2 \mu\left(\varepsilon_{22}-\chi_{22}\right), \quad S_{222}=2 l_{c}^{2} \mu \chi_{22,2}
\end{aligned}
$$

where $\lambda_{s}=0$ and $\mu_{s}=\mu_{c s}=\mu$ are assumed for simplicity. A system of equations is obtained for the two unknowns $\left(\varepsilon_{22}, \chi_{22}\right)$ :

$$
\left\{\begin{array}{l}
\bar{H} \varepsilon_{22,2}+\left(\varepsilon_{22,2}-\chi_{22,2}\right)=0, \\
l_{c}^{2} \chi_{22,22}+\left(\varepsilon_{22}-\chi_{22}\right)=0
\end{array} \text { where } \bar{H}=H /(3 \mu C+H) .\right.
$$

The micro-deformation $\chi_{22}$ is then solution of equation

$$
\chi_{22,222}-\frac{\bar{H}}{l_{c}^{2}(\bar{H}+1)} \chi_{22,2}=0 .
$$

A characteristic length appears in the previous equation:

$$
1 / \omega=l_{c} \sqrt{\left|\frac{\bar{H}+1}{\bar{H}}\right|}
$$

Since $|\bar{H}|$ usually is significantly smaller than 1 (because $|H| \ll \mu$ ), the characteristic length of the problem is larger than $l_{c}$ by a factor of $\sqrt{3 C \mu /|H|}$.

When $H<0$ (softening behaviour), strain localisation will occur in a finite size band. The solution $\chi_{22}$ is sinusoidal with the wave length $\omega$. The localisation zone is an arc of sinus curve for this simple model. Additional boundary conditions are necessary to solve actually the differential equations. They concern the components $u_{i}, \chi_{i j}$ or the dual forces $\left(\sigma_{i j}+s_{i j}\right) n_{j}, S_{i j k} n_{k}$. This model has been applied to strain localisation phenomena in aluminium foams under compression in Forest et al. (2005).

\section{Appendix B. A link between the micromorphic model and the implicit gradient formulation}

It is possible, in the following simplified version of the micromorphic approach, to draw a comparison with a gradient-enhanced approach of softening continua presented in Peerlings et al. (2001). For that purpose, the micromorphic approach is reduced to a microstrain formulation, meaning that only 6 degrees of freedom in addition to the 3 usual translational degrees of freedom, are introduced, namely the 6 components of a symmetric microstrain 
tensor $\chi^{s}$. The microstrain $\chi^{s}$ is the symmetric part of the microdeformation $\chi$. The higher order elasticity laws (15) are then assumed to take the simple form:

$$
\underset{\sim}{s}=b \underset{\sim}{\boldsymbol{e}}=b\left(\underset{\sim}{\varepsilon}-{\underset{\sim}{\boldsymbol{\chi}}}^{s}\right), \quad \underset{\sim}{\boldsymbol{S}}=l_{c}^{2} b \underset{\sim}{\boldsymbol{K}}
$$

where $b$ is a generalised elastic modulus. The relative strain ${\underset{\sim}{s}}^{s}$ measures the deviation of the microstrain from the macrostrain. The gradient of microstrain is $\boldsymbol{K}$. The generalised elasticity tensors have been replaced by a scalar multiplied by the identity. Any kind of classical constitutive equations can be used for the remaining stress $\sigma$ and strain $\boldsymbol{\varepsilon}$. When the simplified elasticity laws (B.1) are introduced in the balance of generalised moment of momentum, the following set of equations is found:

$$
\chi_{i j}^{s}-l_{c}^{2} \Delta \chi_{i j}^{s}=\varepsilon_{i j} .
$$

The Laplacian operator is $\Delta$. The coupling between the classical strain and the microstrain is here clearly visible. This Helmholtz equation for $\chi^{s}$ can be compared to the so-called implicit gradient formulation put forward in Peerlings et al. (2001). In the latter work, a Helmholtz equation is introduced for the so-called nonlocal equivalent strain $\bar{\varepsilon}$. In a similar way, a full nonlocal strain tensor could be defined, solution of Eq. (B.2). Alternatively, such a nonlocal strain can be interpreted as a microstrain tensor in the sense of Eringen (Forest, 2004). The regularisation properties of the implicit gradient model regarding strain localisation phenomena were shown in Peerlings et al. (2001) to be comparable to that of a fully nonlocal medium. The analogy drawn in this appendix between the microstrain model and the implicit gradient model suggests that similar regularising properties could be expected from the micromorphic approach. The examples provided in this work confirm this trend. However, a thorough analysis of the regularising power of the micromorphic model remains to be done.

\section{References}

Andrews, E.W., Gibson, L.J., 2002. On notch-strengthening and crack tip deformation in cellular metals. Mater. Lett. 57, 532-536.

Andrews, E.W., Gioux, G., Onck, P.R., Gibson, L.J., 2001. Size effects in ductile cellular solids: Part II, experimental results. Int. J. Mech. Sci. 43, 701-713.

Ashby, M.F., Evans, A.G., Fleck, N.A., Gibson, L.J., Hutchinson, J.W., Wadley, H.N.G., 2000. Metal Foams: A Design Guide. Butterworth Heinemann.

Badiche, X., Forest, S., Guibert, T., Bienvenu, Y., Bartout, J.-D., Ienny, P., Croset, M., Bernet, H., 2000. Mechanical properties and nonhomogeneous deformation of open-cell nickel foams: application of the mechanics of cellular solids and of porous materials. Mater. Sci. Engrg. A 289, 276-288.

Bart-Smith, H., Bastawros, A.-F., Mumm, D.R., Evans, A.G., Sypeck, D.J., Wadley, H.N.G., 1998. Compressive deformation and yielding mechanisms in cellular Al alloys determined using X-ray tomography and surface strain mapping. Acta Mater. 46, 3583-3592.

Bastawros, A.F., Bart-Smith, H., Evans, A.G., 2000. Experimental analysis of deformation mechanisms in a closed-cell aluminum alloy foam. J. Mech. Phys. Solids 48, 301-322.

Besson, J., 2004. Local Approach to Fracture. Ecole des Mines de Paris-Les Presses.

Besson, J., Abouaf, M., 1989. Microstructural changes in alumina during HIP. Mater. Sci. Engrg. A 109, $37-43$.

Besson, J., Cailletaud, G., Chaboche, J.-L., Forest, S., 2001. Mécanique non linéaire des matériaux. Hermès, France. 445 p.

Blazy, J.-S., Marie-Louise, A., Forest, S., Chastel, Y., Pineau, A., Awade, A., Grolleron, C., Moussy, F., 2004. Deformation and fracture of aluminium foams under proportional and non proportional multi-axial loading: Statistical analysis and size effect. Int. J. Mech. Sci. 46, $217-244$.

Chambon, R., Caillerie, D., Matsuchima, T., 2001. Plastic continuum with microstructure, local second gradient theories for geomaterials. Int. J. Solids Struct. 38, 8503-8527.

Chastel, Y., Hudry, E., Forest, S., Peytour, C., 1999. Mechanical behaviour of aluminium foam for various deformation paths. Experiment and modelling. In: Banhart, J., Ashby, M., Fleck, N. (Eds.), Metal Foams and Porous Metal Structures. Verlag MIT Publishing, pp. $263-268$.

Chen, C., Fleck, N.A., 2002. Size effects in the constrained deformation of metallic foams. J. Mech. Phys. Solids 50, $955-977$.

Cheng, Z.-Q., He, L.-H., 1995. Micropolar elastic fields due to a spherical inclusion. Int. J. Engrg. Sci. 33, 389-397.

Cheng, Z.-Q., He, L.-H., 1997. Micropolar elastic fields due to a circular cylindrical inclusion. Int. J. Engrg. Sci. 35, 659-668.

Chevalier, L., Calloch, S., Hild, F., Marco, Y., 2001. Digital image correlation used to analyze the multiaxial behavior of rubber-like materials. Eur. J. Mech. A Solids 20, 169-187.

de Borst, R., Sluys, L.J., Mühlhaus, H.B., Pamin, J., 1993. Fundamental issues in finite element analyses of localization of deformation. Engrg. Comput. 10, 99-121.

Deshpande, V.S., Fleck, N.A., 2000. Isotropic constitutive models for metallic foams. J. Mech. Phys. Solids 48, $1253-1283$.

Dillard, T., 2004. Comportement mécanique et rupture des mousses de nickel: caractérisation 3D, mesures de champs et simulation numérique. $\mathrm{PhD}$ thesis, Ecole des Mines de Paris.

Dillard, T., NGuyen, F., Maire, E., Forest, S., Bienvenu, Y., Bartout, J.-D., Croset, M., Salvo, L., Dendievel, R., Cloetens, P., 2005. 3D quantitative image analysis of open-cell nickel foams under tension and compression loading using X-ray microtomography. Philos. Mag. A 85, $2147-2175$. 
Doumalin, P., Bornert, M., Crépin, J., 2003. Caractérisation de la répartition de la déformation dans les matériaux hétérogènes. Mécanique et Industries 4, 607-617.

Ebinger, T., Steeb, H., Diebels, S., 2005. Modeling macroscopic extended continua with the aid of numerical homogenization schemes. Comput. Mater. Sci. 32, 337-347.

Eringen, A.C., 1999. Microcontinuum Field Theories. Springer, New York.

Eringen, A.C., Suhubi, E.S., 1964. Nonlinear theory of simple microelastic solids. Int. J. Engrg. Sci. 2, 189-203, 389-404.

Fleck, N.A., Hutchinson, J.W., 2001. A reformulation of strain gradient plasticity. J. Mech. Phys. Solids 49, 2245-2271.

Fleck, N.A., Olurin, O.B., Chen, C., Ashby, M.F., 2001. The effect of hole size upon the strength of metallic and polymeric foams. J. Mech. Phys. Solids 49, 2015-2030.

Forest, S., 2004. Mechanics of generalized continua and heterogeneous materials. Les Presses de l'Ecole des Mines de Paris, 2005.

Forest, S., Blazy, J., Chastel, Y., Moussy, F., 2005. Continuum modelling of strain localization phenomena in metallic foams. J. Mater. Sci. 40, 5903-5910.

Forest, S., Dendievel, R., Canova, G.R., 1999. Estimating the overall properties of heterogeneous Cosserat materials. Modelling Simul. Mater. Sci. Engrg. 7, 829-840.

Forest, S., Lorentz, E., 2004. Localization and regularization. In: Besson, J. (Ed.), Local Approach to Fracture. Ecole des Mines de Paris-Les Presses, pp. 311-373.

Forest, S., Sievert, R., 2003. Elastoviscoplastic constitutive frameworks for generalized continua. Acta Mech. 160, 71-111.

Geers, M.G.D., de Borst, R., Brekelmans, W.A.M., Peerlings, R.H.J., 1998. On the use of local strain fields for the determination of the intrinsic length scale. J. Phys. IV 8 (Pr8), 167-174.

Geers, M.G.D., de Borst, R., Brekelmans, W.A.M., Peerlings, R.H.J., 1999. Validation and internal length scale determination for a gradient damage model: application to short glass-fibre-reinforced polypropylene. Int. J. Solids Struct. 36, 2557-2583.

Hohe, J., Becker, W., 2005. A probabilistic approach to the numerical homogenization of irregular solid foams in the finite strain regime. Int. J. Solids Struct. 42, 3549-3569.

Kesler, O., Gibson, L.J., 2002. Size effects in metallic foam core sandwich beams. Mater. Sci. Engrg. A 326, $228-234$.

Laraba-Abbes, F., Ienny, P., Piques, R., 2003. A new "Tailor-made" methodology for the mechanical behaviour analysis of rubber-like materials: I. Kinematics measurements using a digital speckle extensometry. Polymer 44, 807-820.

Miller, R.E., 2000. A continuum plasticity model for the constitutive and indentation behaviour of foamed metals. Int. J. Mech. Sci. 42, 729-754.

Mindlin, R.D., 1962. Influence of couple-stresses on stress concentrations. Exp. Mech. 3, 1-7.

Mindlin, R.D., 1964. Micro-structure in linear elasticity. Arch. Rational Mech. Anal. 16, 51-78.

Mindlin, R.D., Eshel, N.N., 1968. On first strain gradient theories in linear elasticity. Int. J. Solids Struct. 4, $109-124$.

Neff, P., Forest, S., 2005. A geometrically exact micromorphic model for elastic metallic foams accounting for affine microstructure. Modelling, existence of minimizers, identification of moduli and computational results, J. Elasticity, submitted for publication.

Onck, P.R., 2002. Cosserat modeling of cellular solids. C. R. Mecanique 330, 717-722.

Onck, P.R., Andrews, E.W., Gibson, L.J., 2001. Size effects in ductile cellular solids. Part I: modeling. Int. J. Mech. Sci. 43, 681-699.

Peerlings, R.H.J., Geers, M.G.D., de Borst, R., Brekelmans, W.A.M., 2001. A critical comparison of nonlocal and gradient-enhanced softening continua. Int. J. Solids Struct. 38, 7723-7746.

Pradel, F., Sab, K., 1998. Cosserat modelling of elastic periodic lattice structures. C. R. Acad. Sci. Paris, Ser. IIb 326, 699-704.

Shu, J.Y., 1998. Scale-dependent deformation of porous single crystals. Int. J. Plasticity 14, 1085-1107.

Shu, J.Y., Fleck, N.A., 1999. Strain gradient crystal plasticity: size-dependent deformation of bicrystals. J. Mech. Phys. Solids 47, $297-324$.

Voyiadjis, Z., Abu Al-Rub, K., 2005. Gradient plasticity theory with a variable length scale parameter. Int. J. Solids Struct. 42, $3998-4029$.

Wang, Y., Cuitiño, A.M., 2002. Full-field measurements of heterogeneous deformation patterns on polymeric foams using digital image correlation. Int. J. Solids Struct. 39, 3777-3796.

Xue, Z., Huang, Y., Li, M., 2000. Particle size effect in metallic materials: a study by the theory of mechanism-based strain gradient plasticity. Acta Mater. 50, 149-160.

Zhang, X., Sharma, P., 2005. Inclusions and inhomogeneities in strain gradient elasticity with couple stresses and related problems. Int. J. Solids Struct. 42, 3833-3851. 